\title{
SOX9 is a dose-dependent metastatic fate determinant in melanoma
}

\author{
Xintao Yang ${ }^{1 \dagger}$, Rui Liang ${ }^{1 \dagger}$, Chunxi Liu², Jessica Aijia Liu', May Pui Lai Cheung ${ }^{1}$, Xuelai Liư ${ }^{3}$, On Ying Man", \\ Xin-Yuan Guan ${ }^{5}$, Hong Lok Lung ${ }^{4^{*}}$ and Martin Cheung ${ }^{1 *}$ (D)
}

\begin{abstract}
Background: In this research, we aimed to resolve contradictory results whether SOX9 plays a positive or negative role in melanoma progression and determine whether SOX9 and its closely related member SOX10 share the same or distinct targets in mediating their functions in melanoma.

Methods: Immunofluorescence, TCGA database and QPCR were used to analyze the correlation between the expression patterns and levels of SOX9, SOX10 and NEDD9 in melanoma patient samples. AlamarBlue, transwell invasion and colony formation assays in melanoma cell lines were conducted to investigate the epistatic relationship between SOX10 and NEDD9, as well as the effects of graded SOX9 expression levels. Lung metastasis was determined by tail vein injection assay. Live cell imaging was conducted to monitor dynamics of melanoma migratory behavior. RHOA and RAC1 activation assays measured the activity of Rho GTPases.
\end{abstract}

Results: High SOX9 expression was predominantly detected in patients with distant melanoma metastases whereas SOX10 was present in the different stages of melanoma. Both SOX9 and SOX10 exhibited distinct but overlapping expression patterns with metastatic marker NEDD9. Accordingly, SOX10 was required for NEDD9 expression, which partly mediated its oncogenic functions in melanoma cells. Compensatory upregulation of SOX9 expression in SOX10inhibited melanoma cells reduced growth and migratory capacity, partly due to elevated expression of cyclin-dependent kinase inhibitor p21 and lack of NEDD9 induction. Conversely, opposite phenomenon was observed when SOX9 expression was further elevated to a range of high SOX9 expression levels in metastatic melanoma specimens, and that high levels of SOX9 can restore melanoma progression in the absence of SOX10 both in vitro and in vivo. In addition, overexpression of SOX9 can also promote invasiveness of the parental melanoma cells by modulating the expression of various matrix metalloproteinases. SOX10 or high SOX9 expression regulates melanoma mesenchymal migration through the NEDD9-mediated focal adhesion dynamics and Rho GTPase signaling.

Conclusions: These results unravel NEDD9 as a common target for SOX10 or high SOX9 to partly mediate their oncogenic events, and most importantly, reconcile previous discrepancies that suboptimal level of SOX9 expression is anti-metastatic whereas high level of SOX9 is metastatic in a heterogeneous population of melanoma.

Keywords: SOX9; SOX10, NEDD9, RHOA, RAC1, Melanoma, Metastatic, p21

\footnotetext{
* Correspondence: hllung2@hkbu.edu.hk; mcheung9@hku.hk

${ }^{+}$Xintao Yang and Rui Liang contributed equally to this work.

${ }^{4}$ Department of Biology, Faculty of Science, Hong Kong Baptist University,

Hong Kong, China

${ }^{1}$ School of Biomedical Sciences, Li Ka Shing Faculty of Medicine, The

University of Hong Kong, 21 Sassoon Road, Hong Kong, China

Full list of author information is available at the end of the article
}

(c) The Author(s). 2019 Open Access This article is distributed under the terms of the Creative Commons Attribution 4.0 International License (http://creativecommons.org/licenses/by/4.0/), which permits unrestricted use, distribution, and reproduction in any medium, provided you give appropriate credit to the original author(s) and the source, provide a link to the Creative Commons license, and indicate if changes were made. The Creative Commons Public Domain Dedication waiver (http://creativecommons.org/publicdomain/zero/1.0/) applies to the data made available in this article, unless otherwise stated. 


\section{Background}

Melanoma is one of the most devastating types of human cancer and is the main cause of skin cancer deaths [1]. The aggressiveness of melanoma is due to the combined effects of oncogenic signaling pathways and cancer-relevant transcription factors, resulting in the transformation of neural crest (NC)-derived pigment cells (melanocytes) located in the basal layer of the skin into metastatic melanoma. Melanoma is surgically curable when diagnosed early but its highly metastatic nature considerably worsens the prognosis [2]. Although several treatment regimens to target melanoma harboring the most prevalent BRAF mutation hold great promise with unprecedented response rates, treated patients ultimately develop resistance to therapy after a short period of disease control $[3,4]$. However, intratumoral molecular heterogeneity in a BRAF-mutant melanoma implies a subpopulation of cells develop drug resistance, while another distinct population with different genetic component continues to grow and progress [5]. Therefore, identification and functional characterization of additional gene regulatory pathways to control melanoma growth and metastasis are essential to provide new therapeutic insights.

Our previous studies and others have demonstrated the crucial role of SOXE (Sry (Sex determining gene)-HMG box) E) members of the transcription factor family, SOX9 and SOX10, in NC development [6-8], which belongs to a transient and multipotent stem-like population that gives rise to the peripheral nervous system, craniofacial skeleton and melanocytes [9]. While SOX9 and SOX10 exhibit similar roles in NC specification and migration $[6,10,11]$, the unique expression of SOX10 in embryonic and adult melanocytes dictates its functional requirement for their specification and homeostasis, respectively [12-15]. Consistently, previous studies demonstrated an essential role for SOX10 in the pathogenesis of melanoma in both mice and humans by promoting initiation, proliferation, survival, and invasion [16, 17]. Conversely, SOX9 is expressed in normal human melanocytes but its expression gradually downregulates as melanocytic cells progress from nevi to primary melanoma and are completely absent in the metastatic state, suggesting its negative role in melanoma progression. Indeed, overexpression of SOX9 in both human and mouse melanoma cell lines resulted in inhibition of cell proliferation and tumor growth in xenografts [18]. Another study showed that SOX9 and SOX10 play antagonistic functions in melanoma cells as demonstrated by upregulation of SOX9 expression, which contributed to the pro-apoptotic response induced by SOX10 loss-of-function. These findings indicate that SOX10 could promote melanoma initiation and progression by repressing SOX9 expression, which otherwise would have elicited anti-tumorigenic processes [17]. Moreover, previous studies identified that the melanoma inhibitory activity (MIA) protein was responsible for SOX10-mediated melanoma cell migration and invasion but ectopic expression of MIA could only partially restore the invasive capacity of SOX10-inhibited melanoma cells, suggesting the involvement of other SOX10 target genes [19].

On the contrary, other studies revealed that SOX9 was highly expressed in metastatic melanoma patient samples and contributed to melanoma invasion, suggesting that SOX9 is a negative prognostic factor in advanced melanoma [20, 21]. The discrepancies between the different studies could be attributed to melanoma heterogeneity with distinct expression levels of SOX9 and/or SOX10 in the tumors. Whether they share the same or different downstream targets in mediating melanoma growth and metastasis remain elusive.

Nedd9 (Neural precursor expressed, developmentally down-regulated 9), a member of the Crk-associated substrate (CAS) family of signal transduction proteins, has been demonstrated to function as a scaffolding protein to regulate $\mathrm{NC}$ migration and tumor progression in a variety of cancers including melanoma [22-28]. Our previous studies showed that SOX9 directly transactivates NEDD9 expression to restrict polarized RHOA activity, which is essential for directional migration of mesenchymal NCCs [22]. Likewise, elevation of NEDD9 expression was detected in 30 to $50 \%$ of metastatic melanomas samples and promoted mesenchymal migration of melanoma cells through activation of RAC1 and inhibition of $\mathrm{RHO} / \mathrm{ROCK}$-driven amoeboid movement [29, 30]. Whether NEDD9 expression is also subjected to the transcriptional regulation by SOXE proteins in melanoma remain to be determined.

In this study, using antibodies specific for SOX9, SOX10, and NEDD9, we detected distinct but overlapping expression patterns of SOX10 and NEDD9 in nevi, primary and metastatic melanoma specimens, whereas SOX9 was predominantly and highly expressed in NEDD9 ${ }^{+}$metastatic melanoma in the small intestine and lung. Consistently, as demonstrated in the functional assays, we found NEDD9 expression is regulated by SOX10 and mediates its metastatic functions in melanoma cell lines. When SOX10 expression was silenced, a moderate upregulation of SOX9 expression level was observed and contributed to the anti-metastatic events. We revealed that further increased SOX9 dosage with comparable expression levels to a range of high SOX9 mRNA detected in malignant melanoma specimens could restore the metastatic properties in SOX10 knockdown cells, partly through induction of NEDD9 activity. Lastly, SOX10 or high SOX9 expression mediates melanoma cell migration through the NEDD9-regulated focal adhesion dynamics and Rho GTPase signaling. Taken together, these findings suggest that distinct levels of SOX9 expression determine whether it functions as a suppressor or an inducer of melanoma metastasis.

\section{Methods}

\section{Melanoma specimens}

Surgically procured tumor samples from patients with nevus, primary cutaneous and metastatic melanomas 
were obtained in the Department of Anesthesiology, Zhejiang Cancer Hospital and Department of Pediatric Surgery, the Second Hospital of Hebei Medical University with informed patients' consent for research purposes. All biopsy samples were either fixed with formalin before embedding in the paraffin wax or processed for qPCR analysis.

\section{Constructs and cell lines}

The human SOX9 cDNA was cloned into the lentiviral pWPI vector (Addgene plasmid 12,254). The human NEDD9 cDNA fragment was amplified using pEF-HEF1 as a template and cloned into lentiviral vector pLVX-EF1 $\alpha$-puro (Clontech). The shRNA against the human SOX10 (5'-GACTTCGGC AACGTGGACATT-3') and NEDD9 (5'-GAGACACCA TCTACCAAGTTT-3') were designed based on the principles from The RNAi Consortium (https://www.broadinstitute.org/rnai/public/) and cloned into lentiviral vector pLKO.1-puro. pLKO.1-TRC control was gift from David Root (Addgene plasmid \#10879).

Human epidermal melanocyte (HEMa-LP) was purchased from ThermoFisher and cultured in Medium-254 supplemented with HMGS-2. Human melanoma cell lines A375M, UACC-457, UACC-827, UACC-903 except SK-MEL-28 and human embryonic kidney cell line $293 \mathrm{~T}$ were cultured in DMEM medium with high glucose (Life Technologies) supplemented with $10 \%$ fetal bovine serum (FBS) (BioSera) and $100 \mathrm{U} / \mathrm{ml}$ penicillin-streptomycin (Life Technologies). RPMI-1640 medium (ThermoFisher) was used to culture Me300 kindly provided by D Leung, the Hong Kong University of Science and Technology and SK-MEL-28. Human melanoma cell line WM266-4 was obtained from ATCC and cultured in EMEM medium (Sigma) supplemented with 10\% FBS and $100 \mathrm{U} / \mathrm{ml}$ penicillin-streptomycin. Cell lines were authenticated by cell profiling (AmpFISTR Identifier PCR Amplification kit, Life Technologies).

\section{Lentiviral transduction}

For lentivirus production, $5 \times 10^{6} 293 \mathrm{~T}$ cells were plated in a $100 \mathrm{~mm}$ dish and transfected with a lentiviral expression vector, packaging plasmid psPAX.2 and envelope plasmid pMD2.G using PolyJet ${ }^{\mathrm{tm}}$ (SignaGen). The cell culture medium containing the lentiviral particles was harvested 48 and $72 \mathrm{~h}$ post-transfection and filtered through a $0.22 \mu \mathrm{m}$ filter. $3 \times 10^{5}$ melanoma cells were infected with lentivirus particles expressing cDNA and/or shRNA and cultured in the presence of $8 \mu \mathrm{g} / \mathrm{ml}$ Polybrene (Sigma) for $24 \mathrm{~h}$. After $48 \mathrm{~h}$ transduction, infected melanoma cells were screened in presence of $1 \mu \mathrm{g} / \mathrm{ml}$ puromycin (Life Technologies).

\section{Colony formation assay}

Following puromycin selection of A375M and WM266-4 melanoma cells transduced with lentiviral particles expressing cDNA and/or shRNA, single cell suspension $\left(5 \times 10^{2}\right)$ in complete medium (10\% FBS in DMEM for A375M, 10\% FBS in EMEM for WM266-4) was seeded in each well of a 6-well plate. Plates were incubated at $37^{\circ} \mathrm{C}$ for 1 week for A375M and 2 weeks for WM266-4, during which culture medium was changed every 3 days. Following methanol (Merck) fixation and $0.1 \%$ crystal violet (Sigma) staining, the number of colonies formed in each well was calculated by Quantity One Software (Bio-Rad).

\section{AlamarBlue assay}

Following puromycin selection of A375M and WM266-4 melanoma cells transduced with lentiviral particles expressing CDNA and/or shRNA, single cell suspension $\left(1 \times 10^{3}\right)$ in complete medium (10\% FBS in DMEM for A375M, 10\% FBS in EMEM for WM266-4) was seeded in each well of a 96-well plate and incubated at $37^{\circ} \mathrm{C}$. After $24 \mathrm{~h}$, each well was replaced with $100 \mu \mathrm{L}$ of complete medium containing $10 \%$ AlamarBlue (Life Technologies) and incubated at $37{ }^{\circ} \mathrm{C}$ for $2 \mathrm{~h} .10 \%$ AlamarBlue containing medium in each well was then transferred to a new 96-well plate for measurement of the absorbance reading at $570 \mathrm{~nm}$ and $600 \mathrm{~nm}$. Cells were replaced with a fresh complete medium. $100 \mu \mathrm{L}$ of $10 \%$ AlamarBlue containing medium was added to each well and measured $2 \mathrm{~h}$ post incubation every $24 \mathrm{~h}$ for 4 to 9 days in order to determine the growth curve of the cells with different treatments based on the AlamarBlue absorbance rate between 570 and $600 \mathrm{~nm}$ according to manufacturer's instruction.

\section{Transwell invasion assay}

Following puromycin selection of A375M and WM266-4 melanoma cells transduced with lentiviral particles expressing cDNA and/or shRNA, single cell suspension $\left(5 \times 10^{4}\right)$ in plain medium (DMEM for A375M, EMEM for WM266-4) was seeded on the transparent PET membrane of cell culture insert $(8 \mu \mathrm{m}$, Falcon). For the invasion assay, the membrane was coated with $150 \mathrm{uL}$ of Matrigel $(2.5 \mathrm{mg} / \mathrm{mL}$, Corning) on the ice and gelling at $37^{\circ} \mathrm{C}$ for $6 \mathrm{~h}$ prior to seeding. Cells were allowed to invade through the membrane driven by FBS in the lower chamber for $12 \mathrm{~h}$ (A375M) or $48 \mathrm{~h}$ (WM266-4). Cells failed to invade were removed by the cotton swap. After $100 \%$ methanol fixation and DAPI $(1 \mu \mathrm{g} / \mathrm{ml}$, Sigma) staining, the number of invaded cells was counted in 15 random fields within the membrane under an inverted fluorescence microscope.

\section{Western blot}

Cells were washed twice with cold phosphate-buffered saline (PBS) and lysed in RIPA buffer $(150 \mathrm{mM} \mathrm{NaCl}, 1 \mathrm{mM}$ EDTA, 1\% NP40, 0.5\% Sodium deoxycholate, 0.1\% SDS, 50 $\mathrm{mM}$ Tris- $\mathrm{HCl}, \mathrm{pH} 7.5)$ supplemented with $1 \%$ protease and phosphatase inhibitor cocktail (ThermoFisher). Proteins were separated by SDS-PAGE using the Bio-Rad system under reducing conditions. Membranes were probed with 
antibodies against SOX9 (H-90, Santa Cruz), SOX10 (N-20, Santa Cruz), NEDD9 (Clone 2G9, Abcam) and GAPDH (FL-335, Santa Cruz) for overnight at $4{ }^{\circ} \mathrm{C}$ and then incubated with appropriate horseradish peroxidase-conjugated goat anti-rabbit, rabbit anti-mouse and donkey anti-goat antibodies (at 1:2000, Dako) at room temperature for $1 \mathrm{~h}$. After incubation with ECL substrate (WesternBright, Advansta) for 1-3 min, blots were exposed to X-ray film (FujiFilm Super RX) at different times to obtain the optimal intensity of the protein bands which were analyzed by ImageJ.

\section{Quantitative polymerase chain reaction (qPCR)}

Total RNA was extracted using MiniBEST Universal RNA Extraction Kit (Takara) and reverse transcribed for cDNA synthesis using PrimeScript RT Master Mix (Takara). All reactions including non-template controls were performed in triplicate on StepOnePlus Real-time PCR system (Applied Biosystem) using SYBR Premix Ex Taq II (Takara). Human 36B4 was used for normalization. List of primers for detection of gene expression is listed below.

\begin{tabular}{|c|c|c|c|}
\hline Gene & Species & $\begin{array}{l}\text { Probe length } \\
(\mathrm{bp})\end{array}$ & Primers $\left(5^{\prime}-3^{\prime}\right)$ \\
\hline \multirow[t]{2}{*}{ SOX10 } & Human & 83 & For: GACCAGTACCCGCACCTG \\
\hline & & & Rev: CGCTTGTCACTTTCGTTCAG \\
\hline \multirow[t]{2}{*}{ SOX9 } & Human & 102 & For: ACACACAGCTCACTCGACCTTG \\
\hline & & & Rev: GGAATTCTGGTTGGTCCTCTCTT \\
\hline \multirow[t]{2}{*}{ NEDD9 } & Human & 159 & For: ATGTCCACGTCTTCCACCTCC \\
\hline & & & Rev: AGTGACCAGTGCCATTAGGCTG \\
\hline \multirow[t]{2}{*}{$36 B 4$} & Human & 101 & $\begin{array}{l}\text { For: GTGATGTGCAGCTGATCAA } \\
\text { GACT }\end{array}$ \\
\hline & & & Rev: GAAGACCAGCCCAAAGGAGA \\
\hline \multirow[t]{2}{*}{ MMP1 } & Human & 111 & For: AGGTCTCTGAGGGTCAAGCA \\
\hline & & & Rev: CTGGTTGAAAAGCATGAGCA \\
\hline \multirow[t]{2}{*}{ MMP2 } & Human & 148 & For: AAGAAGTAGCTGTGACCGCC \\
\hline & & & Rev: TTGCTGGAGACAAATTCTGG \\
\hline \multirow[t]{2}{*}{ MMP3 } & Human & 138 & For: ATTCCATGGAGCCAGGCTITC \\
\hline & & & $\begin{array}{l}\text { Rev: CATTTGGGTCAAACTCCAA } \\
\text { CTGTG }\end{array}$ \\
\hline \multirow[t]{2}{*}{ MMP7 } & Human & 158 & For: GAGTGAGCTACAGTGGGAACA \\
\hline & & & Rev: CTATGACGCGGGAGTTTAACAT \\
\hline \multirow[t]{2}{*}{ MMP8 } & Human & 154 & For: TCTGCAAGGTTATCCCAAGG \\
\hline & & & Rev: ACCTGGCTCCATGAATTGTC \\
\hline \multirow[t]{2}{*}{ MMP23 } & Human & 144 & For: CCAGAAGATCCTCCACAAGA \\
\hline & & & Rev: CAGGTGTAGGTGCCCTCATT \\
\hline
\end{tabular}

\section{Luciferase reporter assay}

A375M and WM266-4 melanoma cells were transfected with FireFly luciferase reporter vector driven by human NEDD9 proximal promoter $(\sim 1 \mathrm{~kb})$ and Renilla luciferase reporter vector using Polyjet transfection reagent based on manufacturer's protocol. Cells were harvested and lysed $48 \mathrm{~h}$ post-transfection. The cell lysate was measured by PerkinElmer Victor 3 Multi-label Plate Reader using Dual-Luciferase Reporter Assay System (Promega) according to the manufacturer's instructions. The luminescence signal of the Renilla luciferase reporter activity was used for normalization of FireFly luciferase reporter activity.

\section{Chromatin immunoprecipitation}

A375M melanoma cells were transduced with SOX10 or SOX9-overexpressing lentivirus. A total of $6 \times 10^{6}$ cells for each treatment were fixed by $1 \%$ formaldehyde and lysed, then digested using micrococcal nuclease according to the manufacturer's protocol (Pierce Agarose ChIP Kit, 26,156, Thermofisher). Supernatant was collected and sonicated for $6 \times 30 \mathrm{~s}$ in a Bioruptor sonicator (Diagenode). The target size of chromatin fragments ranging from 400 bp to 600 bp was confirmed by $2 \%$ agarose gel electrophoresis. Chromatin fragments were immunoprecipitated by using normal rabbit IgG control (Thermofisher), $2 \mu \mathrm{g}$ anti-SOX10 antibody (ChIP grade, sc-17,342X, Santa Cruz) or $2 \mu \mathrm{g}$ anti-SOX9 antibody (ChIP grade, AB5535, Millipore) at $4{ }^{\circ} \mathrm{C}$ overnight. 20uL of ChIP grade Protein A/G Plus Agarose (Thermofisher) was added into the chromatin-antibody mixture and incubated at $4{ }^{\circ} \mathrm{C}$ for $2 \mathrm{~h}$. DNA fragments were then purified and recovered based on manufacturer's instruction, followed by 40 cycles of quantitative PCR. Primers used for amplification of fragments covering SOX binding motif (AAACAAA) are: 5' - GGAGGGCCACTAGC TAGAGA-3' and 5'-GCCTCCAAGAGATCTAGAT AAC-3'. Primers targeting non-SOX binding motif are 5'- TTTTCGCCTCACTGCTCTGT-3' and 5'- GGCT GGCATTTCTAGCTCCA-3'. Data were analyzed and presented as the fold enrichment relative to IgG control.

\section{Time-lapse imaging of melanoma cell migration in a wound-healing assay}

A375 melanoma cells transduced with Lifeact-mCherry together with different constructs were cultured in a $10 \mathrm{~cm}^{2}$ dish until $90 \%$ confluent. A migration gap of approximately $1 \mathrm{~mm}$ was then created by introducing a "scratch" to the adherent layer of cultured cells using a sterile $200 \mu \mathrm{L}$ pipette tip. At this point, half of the culture medium was removed and replaced with fresh medium to reduce the number of cells introduced into suspension reattaching to the cell-free zone during experimentation. Time-lapse imaging of melanoma cells 
migrating to the wound was performed on a Perkin Elmer Widefield imaging microscope equipped with an incubator capable of maintaining $37^{\circ} \mathrm{C}$ temperature, $95 \%$ relative humidity and $5 \% \mathrm{CO} 2$. Images were acquired with 10X objective and collected every $5 \mathrm{~min}$ for a total period of $7.5 \mathrm{~h}$ at one time. Images were analyzed by Image $J$ software. The cell speed was determined by the total distance traveled divided by the time.

\section{RHOA and RAC1-activation assay}

Detection of RHOA and RAC1 activity was performed by RHOA and RAC1 Pull-down Activation Assay Biochem Kits (Cytoskeleton) based on manufacturer's protocol. Melanoma cells at $80 \%$ confluence were washed twice with cold PBS and lysed with cold Cell Lysis Buffer supplied with 1\% Protease and Phosphatase Inhibitor Cocktail for $3 \mathrm{~min}$ on ice. Cell lysates were then centrifuged at $10,000 \mathrm{~g}$ for $3 \mathrm{~min}$. The supernatant $(600 \mu \mathrm{g}) \quad$ was then immediately incubated with Rhotekin-RBD $(50 \mu \mathrm{g})$ or PAK-PBD $(20 \mu \mathrm{g})$ beads at $4{ }^{\circ} \mathrm{C}$ overnight. Active RHOA and RAC1 were pull downed by the Rhotekin-RBD and PAK-PBD beads respectively, which were washed six times by wash buffer followed by western blotting using mouse monoclonal antibodies against RHOA (Santa Cruz) and RAC1 (Santa Cruz).

\section{Immunofluorescence}

After deparaffinization and rehydration, the paraffin melanoma sections $(5 \mu \mathrm{m})$ were subjected to antigen retrieval by boiling in Target Retrieval Solution (Citrate $\mathrm{pH}$ 6.0, Dako) for $10 \mathrm{~min}$ and cooled down to room temperature for $30 \mathrm{~min}$. Sections were blocked in $1 \%$ normal donkey serum with $0.1 \%$ TritonX-100 and followed by $4{ }^{\circ} \mathrm{C}$ overnight incubation of primary antibodies (diluted in 1\% normal donkey serum PBS) against SOX9 (1:100, H-90, Santa Cruz), SOX10 (1:200, N-20, Santa Cruz) and NEDD9 (1:1000, Clone 2G9, Abcam). Anti-rabbit, anti-mouse and anti-goat antibodies conjugated with Alexa-488, Alexa-555 and Alexa-647 were applied as secondary antibodies. For staining of focal adhesion and stress fiber, $1 \times 10^{4}$ melanoma cells were seeded onto sterile coverslips in 24-well plate $24 \mathrm{~h}$ prior to $4 \%$ formaldehyde fixation on ice for $30 \mathrm{~min}$. Cells on coverslip were blocked by $1 \%$ bovine serum albumin with $0.03 \%$ TritonX-100 in PBS, followed by $4{ }^{\circ} \mathrm{C}$ overnight incubation of anti-Vinculin (1:500, Invitrogen) and then one-hour room temperature incubation of Alexa Fluor 546 Phalloidin (1:500, Cytoskeleton). DAPI $(1 \mu \mathrm{g} / \mathrm{ml}$, Sigma) was used as a nuclear counter-stain for both immunostained sections and cells on coverslips. Fluorescence images were captured using Carl Zeiss LSM 780 confocal microscope and analyzed by ZEN 2011 and MetaMorph software in the Faculty Core
Facility, Li Ka Shing Faculty of Medicine, University of Hong Kong.

\section{In vivo pulmonary metastasis assay}

The following mouse experimentation was approved by the Committee on the Use of Live Animals in Teaching and Research (CULATR), University of Hong Kong (CULATR no: 4005-16).

After lentiviral transduction and puromycin selection, a $100 \mu \mathrm{L}$ single cell suspension containing $1 \times 10^{6}$ A375M cells in plain DMEM was injected into the tail vein of 7-week-old female NOD-SCID mice. Four weeks post-injection, mice were anesthetized before intraperitoneal injection of $100 \mathrm{uL}$ of sterile D-Luciferin firefly potassium salt solution $(30 \mathrm{mg} / \mathrm{mL})$. The tumor cells colonized in the lung as reflected by bioluminescent signals were acquired for $4 \mathrm{~min}$ in vivo imaging using Xenogen IVIS 200. Regions of interest (ROI) were manually selected, and the results were quantified as the average radiance of photons emitted per second and area by using the Living Image software (Xenogen, Alameda, CA). After quantification, mice were sacrificed and the lung nodules were counted.

\section{Statistical analysis}

The experiments were performed at least three times independently. The statistical data were expressed as Mean \pm SD (SD $=$ standard deviation of the mean values of each independent experiments). Student's t-test and two-way analysis of variance (ANOVA) were used to determine the confidence levels for group comparison. Values are statistically significant at $\mathrm{p}^{*}<0.05$; ${ }^{* * *} p<0.01$; ***** $p<0.001$.

\section{Results}

Positive correlation of expression between SOXE and NEDD9 in melanoma patient specimens

Our recent studies demonstrated that NEDD9 is a direct transcriptional target of SOX9 in mediating neural crest delamination [22]. In addition, NEDD9, SOX9, and SOX10 have been shown to be crucial for human melanoma metastasis [20, 28]. Whether SOX9 and/or SOX10 exhibit a similar regulatory relationship with NEDD9 in melanoma has not yet been examined. To address this issue, we first performed comparative expression study of these factors on tissue sections from Chinese patients with benign melanocytic nevus, primary dermal and metastatic melanomas using antibodies specific for SOX9, SOX10, and NEDD9 [17, 31]. Immunofluorescence staining showed that NEDD9 was localized in the cytoplasm and co-expressed with most, if not all, of SOX $10^{+}$pigmented nevus and primary melanomas, whereas SOX9 was barely detectable (Fig. 1a-c). In agreement with this, we performed qPCR for $S O X 9$ on a 


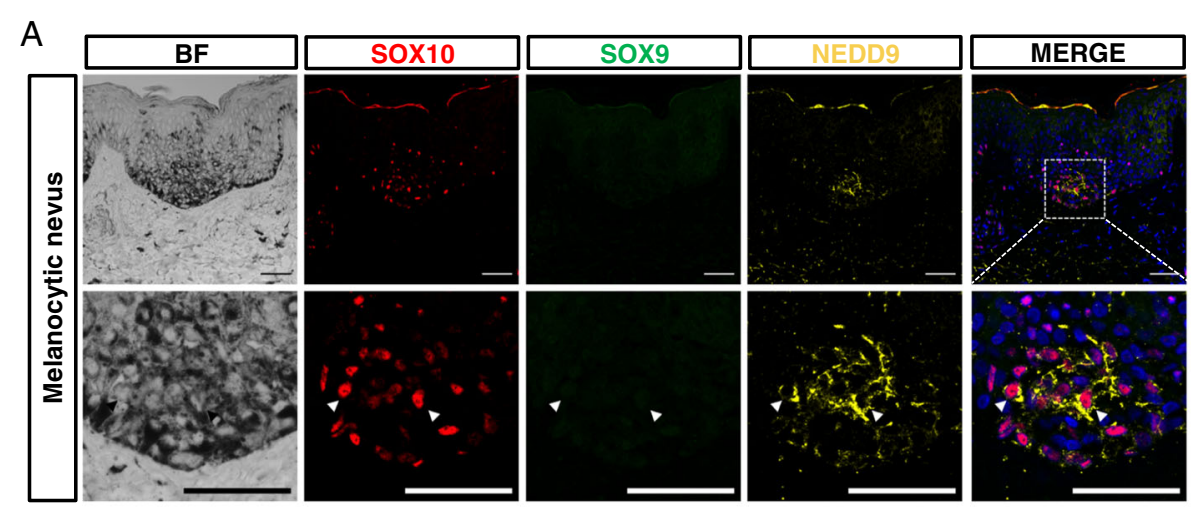

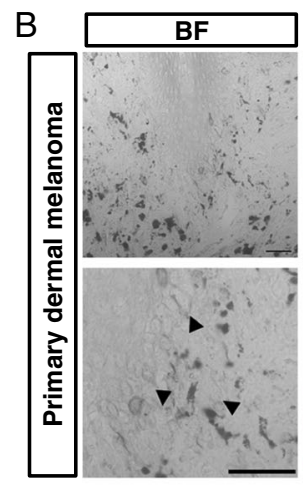

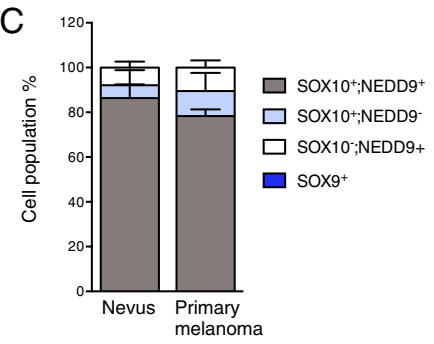

D
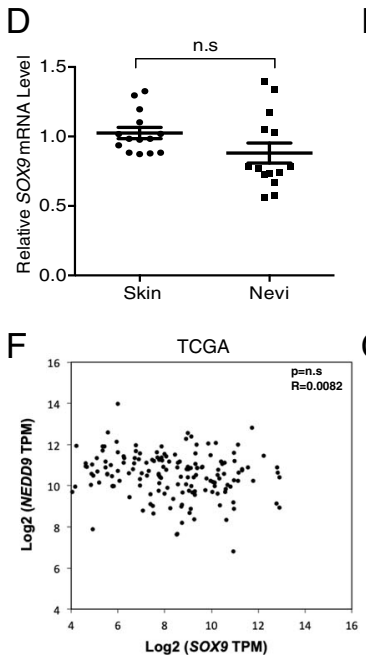
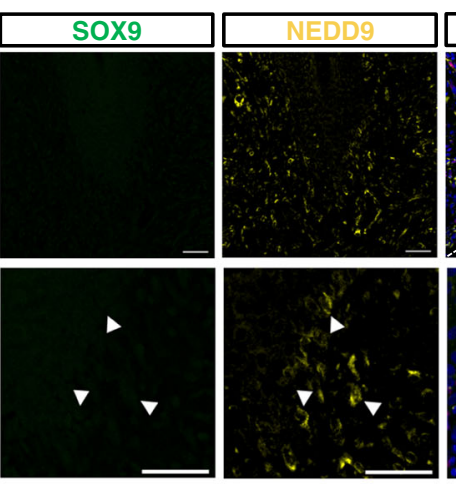

E
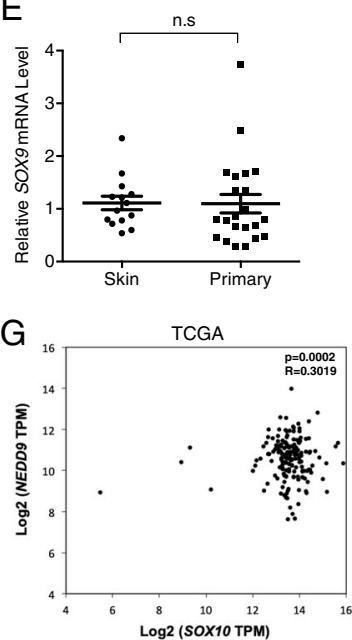

Fig. 1 Co-expression of SOX10 and NEDD9 but not SOX9 in melanocytic nevi and primary dermal melanomas. a, b Representative images showing immunofluorescence for SOX10, SOX9, and NEDD9 in the skin sections of patients with benign melanocytic nevus (a) and primary dermal melanoma (b). White arrowheads indicate cells coexpressing SOX10 and NEDD9 but not SOX9. The dotted white box in the merged image indicates the magnified region with separate color channels shown in the lower panels. Cell nuclei were counterstained by DAPI (blue). Scale bars: $10 \mu \mathrm{m}$. c Quantification of the number of cells positive for the indicated markers in 12 melanocytic nevi and 14 primary dermal melanoma samples. $\mathbf{d}$, e qPCR analysis of SOX9 expression in 14 healthy skin controls, 14 melanocytic nevi, and 22 primary melanoma samples. $\mathbf{f}$ Correlation expression analysis between SOX9 and NEDD9; SOX10 and NEDD9 (g) in melanoma patient samples obtained from Skin Cutaneous Melanoma dataset in TCGA (173 patients). Error bars represent mean \pm SD. n.s, non-significant. The $P$-value and Pearson correlation coefficient are denoted on top

cohort of melanocytic nevi and primary melanomas and the majority of which remained at the basal level of expression (Fig. 1d, e). Similarly, analyzing the Cancer Genome Atlas (TCGA) dataset from 173 patients with primary cutaneous melanomas revealed no significant correlation of expression between SOX9 and NEDD9 whereas we observed a moderate but significant correlation of expression between SOX10 and NEDD9 (Fig. 1f, g). In contrast, SOX9 expression began to express in a subset of NEDD9 $^{+}$melanoma cells, which had 

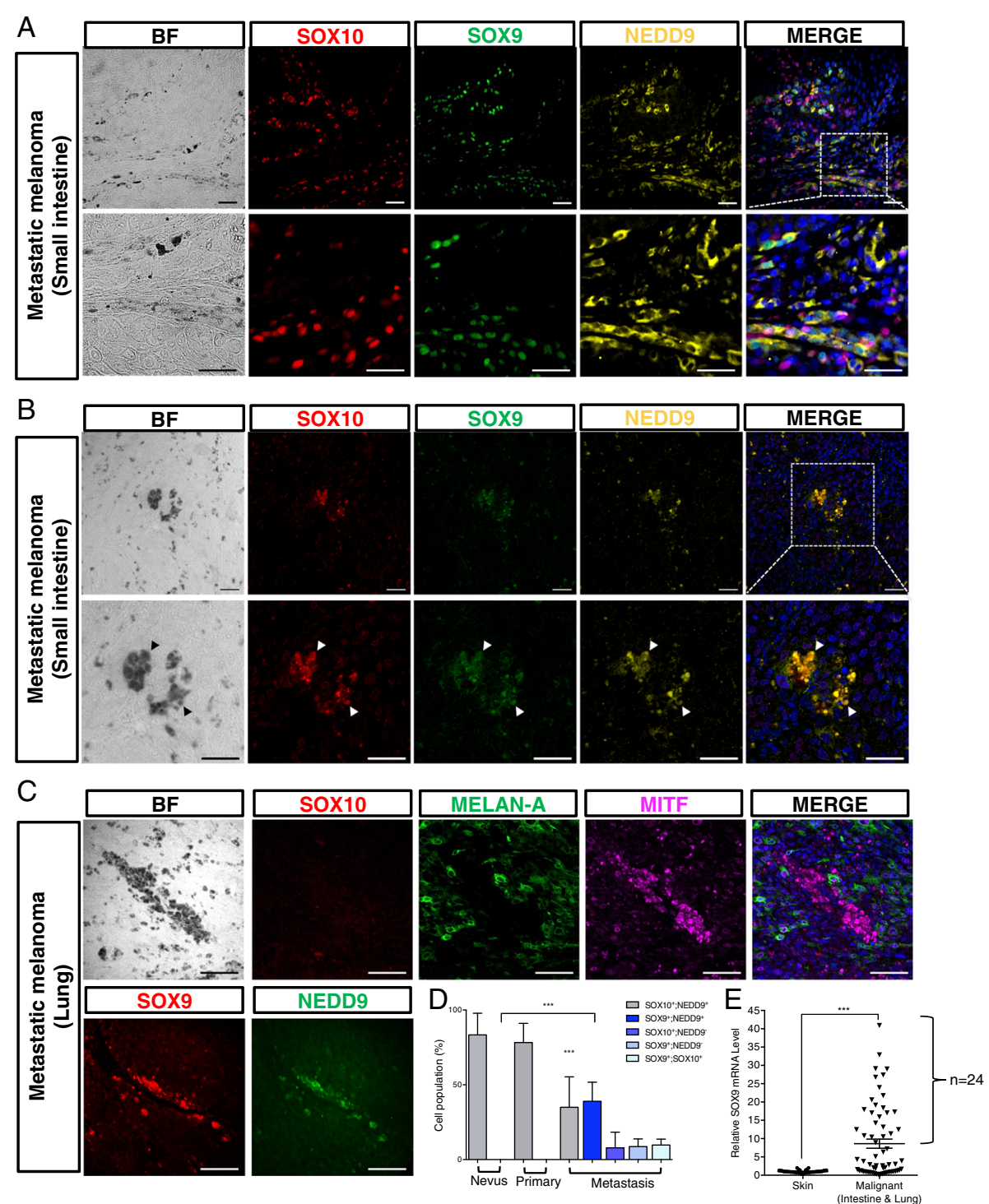

Fig. 2 SOX9 expression in metastatic melanomas. a, b Representative images showing immunofluorescence for SOX9, SOX10, and NEDD9 in the cross-section of the intestinal metastatic melanoma from two patients. b Arrowheads indicate pigmented melanoma cells co-expressing SOX9, SOX10, and NEDD9. The dotted white box in the merged image indicates the magnified region with separate color channels shown in the lower panels. c Representative images showing immunofluorescence on consecutive lung sections with metastatic melanoma for SOX10, MELAN-A, MITF, as well as SOX9 and NEDD9. Cell nuclei were counterstained with DAPI (blue). Scale bars: $10 \mu \mathrm{m}$. d Quantification of the number of cells positive for the indicated markers in 12 melanocytic nevi, 14 primary dermal melanomas and 25 metastatic melanomas. e The amount of SOX9 transcripts was measured by qRT-PCR in 22 healthy skin controls, metastatic melanoma specimens from the intestine $(n=37)$ and lung ( $n=27)$. Error bars represent mean \pm SD. Student's $t$-test, ${ }^{* * *} p<0.001$

metastasized to the small intestine and another subset of NEDD9 $^{+}$cells exhibited SOX10 expression (Fig. 2a, d), whereas we detected co-expression of SOX10, SOX9, and NEDD9 in another patient with intestinal melanomas (Fig. 2b, d). In another sample, we found no SOX10 expression in lung metastases where subset of pigmented cells exhibited co-expression of SOX9 and NEDD9 (Fig. 2c, d). Although these cells were negative for a melanoma marker MELAN-A [32], they were positive with a diagnostic marker for metastatic melanoma MITF
[33] (Fig. 2c). Moreover, there was no strict correlation between the patterns of SOX9, SOX10, and NEDD9 expression and distribution of pigmented melanomas in all examined stages (Fig. 1a, b, and Fig. 2a-c). Taken together, our immunofluorescence studies demonstrate a positive correlation between NEDD9 and SOX10 expression in human tissue biopsies from different stages of melanoma progression including common acquired nevi, primary melanoma, and metastases, while SOX9 expression appears to associate with a more invasive and 
metastatic phenotype. Indeed, $\mathrm{qPCR}$ analysis revealed that elevated SOX9 expression was frequently detected in lung and intestine metastatic melanomas compared to the control skin samples from healthy subjects (Fig. 2e). These results demonstrate that high SOX9 expression is predominantly associated with metastatic melanomas.

\section{Upregulated level of SOX9 expression contributes to cell growth arrest, reduced migratory capacity and colony formation activity in SOX10 knockdown melanoma cells} The predominant association of SOX10 and NEDD9 but not SOX9 expression in melanoma specimens is further supported by co-expression of these two factors at different levels in a series of malignant melanoma cell lines (Fig. 3a, b). SOX10 levels were higher in all melanoma cell lines than in normal human melanocytes (HEMa-LP), whereas SOX9 expression was low in all these cell lines. These prompted us to examine whether SOX10 regulates NEDD9 expression in two metastatic melanoma cell lines (A375M and WM266-4) harboring mutated BRAF, which express high levels of SOX10 and NEDD9 expression (Fig. $3 \mathrm{a}, \mathrm{b})$. We first analyzed NEDD9 expression in these cell lines transduced with lentiviral-shRNA-scramble control or shRNA-mediated knockdown of SOX10 (SOX10 KD). We observed a significant reduction of NEDD9 transcripts in SOX10 KD compared to control, suggesting that NEDD9 expression could be regulated by SOX10 in melanoma cells. In contrast, SOX9 expression was significantly upregulated in SOX10 KD (Fig. 3c), consistent with previous observations that SOX10 normally suppressed SOX9 expression which otherwise would have elicited a pro-apoptotic response in melanoma cells [17]. Importantly, the upregulated levels of SOX9 expression in SOX10 KD A375 (1.5 to 2.6 fold) and WM266-4 (1.5 to 3.4 fold) are clinical relevant as they fall within the range of SOX9 expression levels detected in some specimens of primary melanoma (1.3 to 3.7 fold) (Fig. 1e and Fig. 3c). There was no significant difference in the degree of reduced NEDD9 expression between SOX10 KD and SOX9 KD + SOX10 $\mathrm{KD}$, suggesting that upregulated level of SOX9 expression did not contribute to the reduction of NEDD9 expression in SOX10 KD cells (Fig. 3d). To further expand the studies on the anti-tumorigenic effects of increased SOX9 levels in SOX10 KD, we performed SOX9 KD in both SOX10 KD A375M and WM266-4 melanoma cells and compared the effects with $S O X 10 \mathrm{KD}$ alone and control on cell proliferation, invasion and oncogenicity using alamarBlue, transwell and colony formation assays respectively. While SOX10 KD resulted in a marked reduction of cell growth (Fig. 3e), invasive capacity (Fig. 3f, g), and colony formation (Fig. 3h,i), SOX9 KD partly restored these properties in SOX10 KD cells (Fig. 3e-i). These results further confirm that the upregulated $S O X 9$ expression contributes to the anti-tumorigenic and anti-metastatic effects of SOX10 KD melanoma cells.

On the other hand, previous studies showed that either overexpression of SOX9 alone or upregulation of SOX9 expression in SOX10 KD caused cell cycle arrest through an increase in cyclin-dependent kinase inhibitor p21 protein expression in melanoma cell lines $[16,18]$. These results prompted us to further examine whether the levels of SOX9 expression determine the degree of p21 induction. In agreement with previous observations, we observed a pronounced elevation of p21 protein upon SOX10 KD in both A375M and WM266-4 melanoma cells compared to the scramble control, whereas increased p21 expression was alleviated by SOX9 KD (Fig. 3j), indicating that upregulation of endogenous SOX9 by SOX10 $\mathrm{KD}$ is required for the induction of p21 expression. We then further applied the amount of lentiviral vector expressing SOX9 (SOX9 OE) at two different titers $(100 \mu \mathrm{L}$ and $200 \mu \mathrm{L}$ ) in the SOX10 KD cells, and that resulted in increased SOX9 mRNA levels by 12 and 33-fold in A375, and 13 to 44-fold in WM266-4 respectively (Fig. 5a). These levels fall within the range of high SOX9 expression being detected in the metastatic melanoma samples (10 to 44-fold vs normal skin, Fig. 2e). The results showed that the progressive elevation of SOX9 expression levels caused a gradual reduction of p21 expression (Fig. 3j). Moreover, we obtained a greater degree of reduction in p21 expression level at the highest dose $(200 \mu \mathrm{L})$ of SOX9 OE in parental cells (Fig. 3j). Altogether, these data demonstrate that in the absence of SOX10, the upregulated or low levels of endogenous SOX9 expression promote p21 expression whereas high levels of SOX9 could inhibit p21 expression and may enhance cell growth.

\section{NEDD9 functions downstream of SOX10}

The downregulation of NEDD9 expression in SOX10 KD prompted us to examine whether $N E D D 9$ knockdown (NEDD9 KD) would exhibit a similar functional outcome as in SOX10 KD. qPCR and Western blot analysis of A375M and WM266-4 cells treated with shRNA-NEDD9 showed a significant reduction in the levels of NEDD9 RNA and protein of both parental and phosphorylated forms compared with the scramble control respectively (Fig. 4a,b). In vitro functional studies further showed that NEDD9 KD caused a marked reduction of cell growth (Fig. 4c), invasive behavior (Fig. 4d,e) and colony formation capacity (Fig. 4f,g) as observed in SOX10 KD (Fig. $4 \mathrm{c}-\mathrm{g}$ ), suggesting that NEDD9 is required for proliferation, invasion and oncogenicity of melanoma cells. Importantly, NEDD9 KD did not significantly alter the transcript and protein levels of SOX9 and SOX10 (Fig. 4a,b). These results suggest that NEDD9 could function downstream of SOX10 to mediate its tumorigenic effects. Indeed, overexpression of NEDD9 gene (NEDD9 OE) restored cell 
A

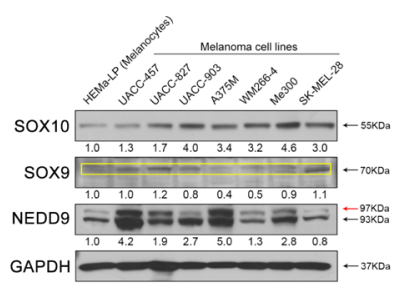

C

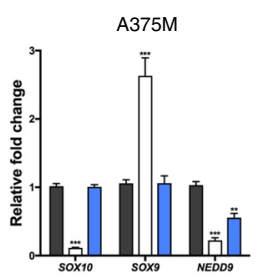

E

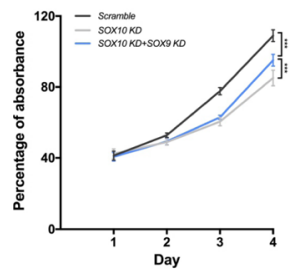

G

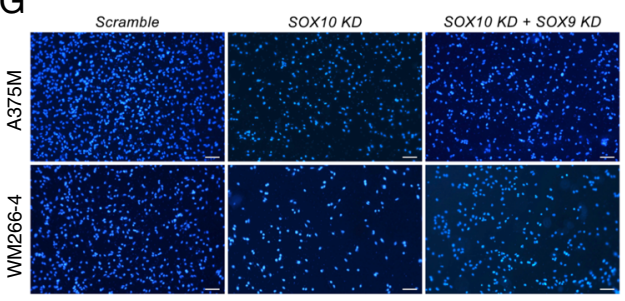

B

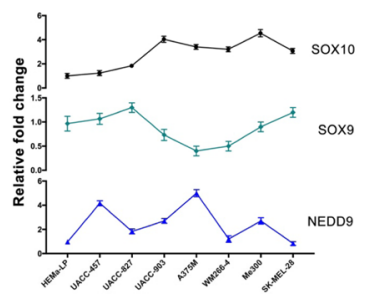

D

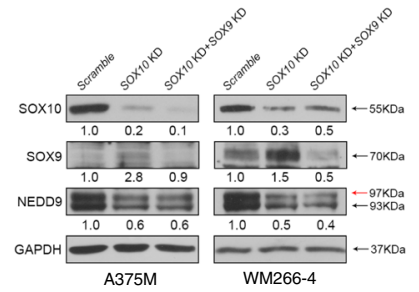

F
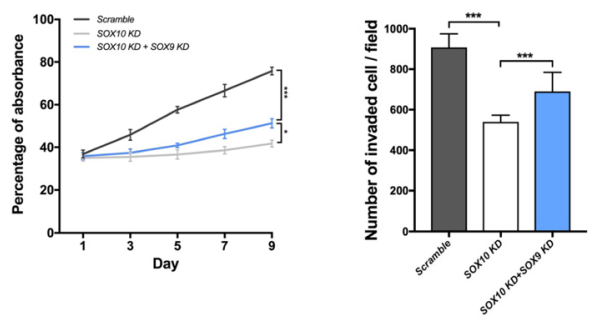

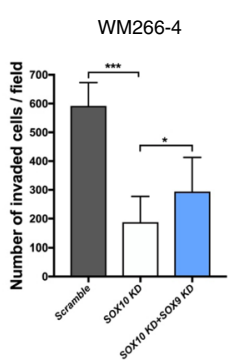

$\mathrm{H}$
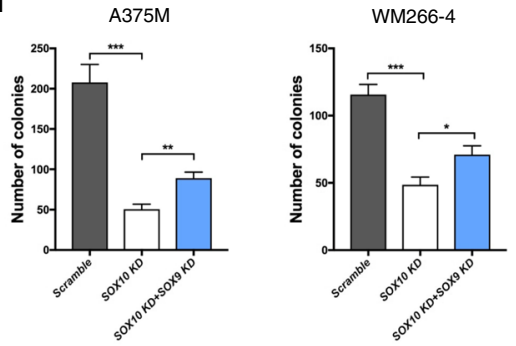

I

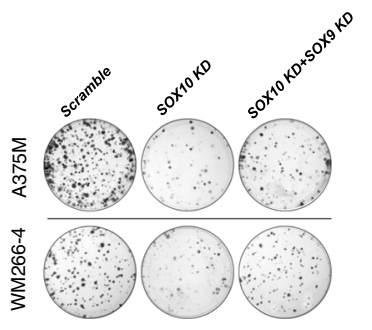

J

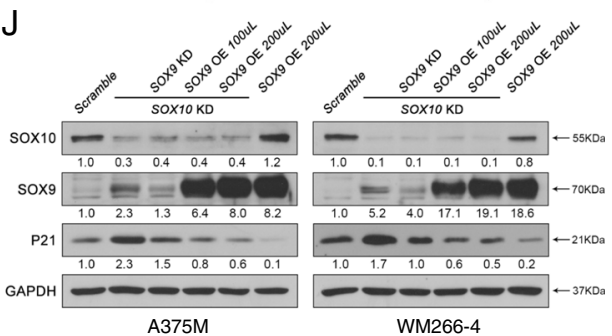

Fig. 3 Upregulated or low level of SOX9 expression contributes to the anti-metastatic/anti-oncogenic activities of SOX10 knockdown (KD) melanoma cells. a Expression of SOX10, SOX9, and NEDD9 in human melanocytes (HEMa-LP), and a panel of metastatic melanoma cell lines. GAPDH was used as a loading control. The yellow box indicates protein bands corresponding to the size of SOX9. The red arrow indicates a phosphorylated form of NEDD9. The intensity of protein bands in arbitrary units for SOX10, SOX9, and NEDD9 in each melanoma cell line is relative to HEMa-LP which is set to 1 as a reference. $\mathbf{b}$ Line plots represent the intensity of protein bands shown in (a). c qRT-PCR analysis of SOX10, SOX9 and NEDD9 transcript levels in A375M and WM266-4 cells treated with scramble control, SOX10 KD and NEDD9 KD. Data represent fold change normalized to scramble control and the average of three independent assays. $\mathbf{d}$ Western blot analysis of SOX9, SOX10 and NEDD9 protein levels in each cell line transduced with scramble control, SOX10 KD and SOX10 KD + SOX9 KD. GAPDH serves as a loading control. The red arrow indicates a phosphorylated form of NEDD9. AlamarBlue (e), transwell invasion (f, $\mathbf{g})$ and colony formation assays $(\mathbf{h}, \mathbf{i})$ of each cell line treated with scramble control, SOX10 KD and SOX10 KD + SOX9 KD. $\mathbf{g}$ DAPI images of transwell invasion of melanoma cells treated with the indicated constructs. Scale bars: $100 \mu \mathrm{M}$ (i) Representative images showing crystal violet stained colonies formed from A375M and WM266-4 cells treated with scramble control, SOX10 KD and SOX10 KD + SOX9 KD. j Western blot analysis of SOX10, SOX9 and p21 protein levels in each cell line transduced with scramble control, SOX10 KD alone, SOX10 KD + SOX9 KD, two different volume (100 $\mu \mathrm{L}, 200 \mu \mathrm{L})$ of lentiviruses encoding SOX9 gene (SOX9 OE) in SOX10 KD and maximum dose of SOX9 OE in parental cells. GAPDH serves as a loading control. Error bars represent mean \pm SD of three independent experiments. ${ }^{*} p<0.05,{ }^{* *} p<0.01,{ }^{* * *} p<0.001$ 

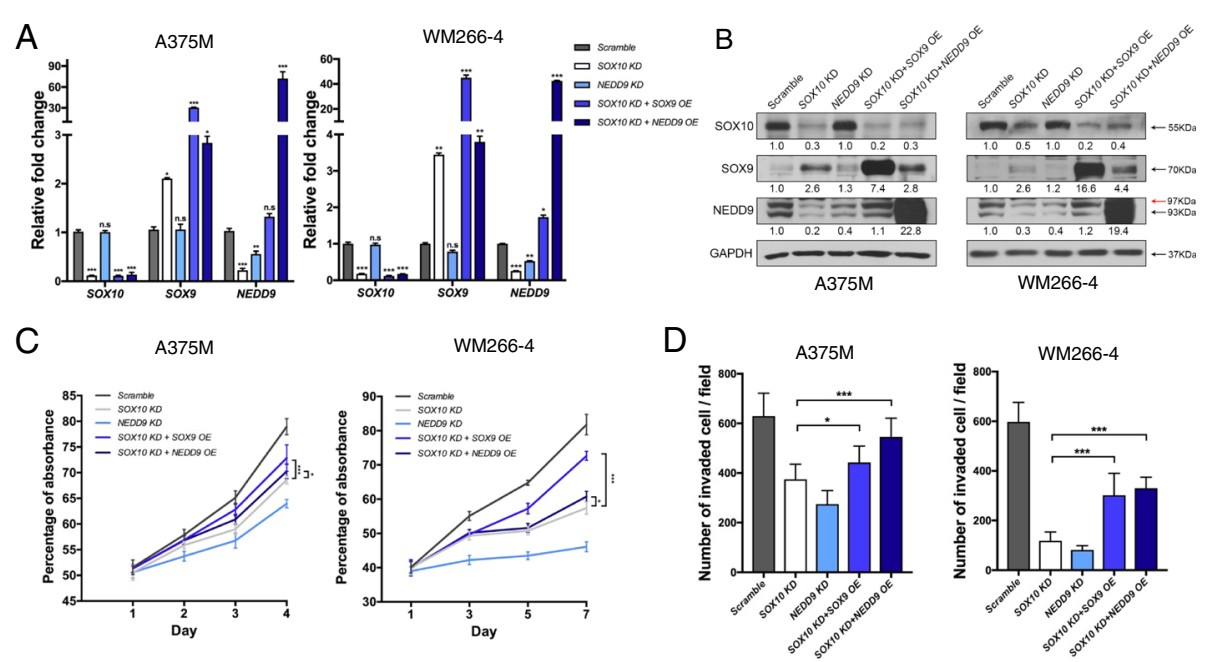

$\mathrm{E}$
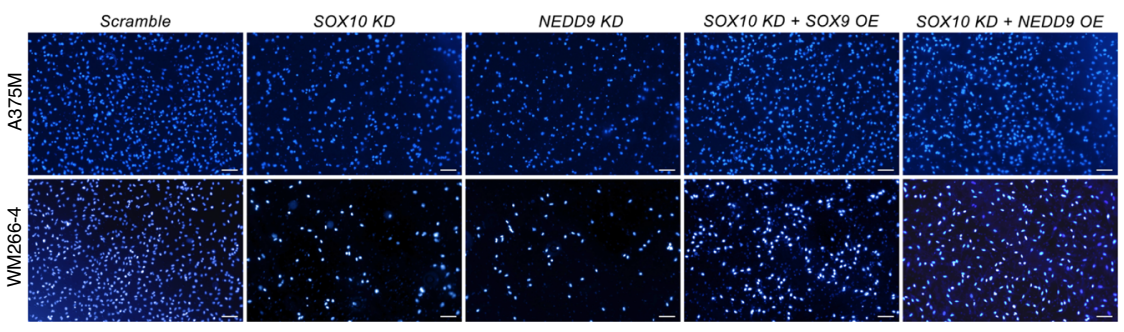

$\mathrm{F}$
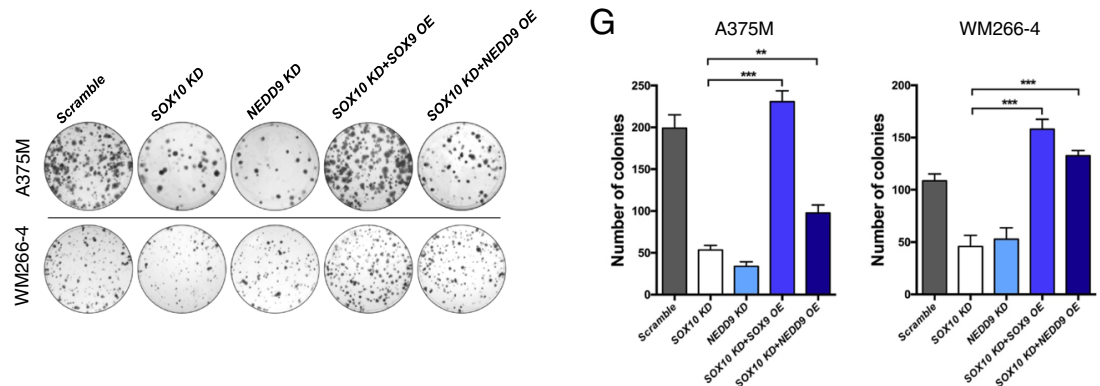

Fig. 4 Overexpression of SOX9 and NEDD9 restore the oncogenic features of SOX10 KD melanoma cells. QRT-PCR (a) and Western blot (b) analysis for the expression levels of SOX10, SOX9 and NEDD9 in A375M and WM266-4 cell lines treated with the indicated constructs. Data are fold change normalized to scramble control and the average of three independent assays. The red arrow indicates the phosphorylated form of NEDD9. GAPDH serves as a loading control. AlamarBlue (c) and transwell invasion assays ( $\mathbf{d}$ ) of each cell line treated with the indicated constructs. e DAPI images of transwell invasion of melanoma cells treated with the indicated constructs. Scale bars: $100 \mu \mathrm{M}$. f Representative images of crystal violet stained A375M and WM266-4 clones subjected to different treatments. $\mathbf{g}$ Quantification of the number of A375M and WM266-4 colonies treated with the indicated constructs. Error bars represent mean \pm SD of three independent experiments. ${ }^{*} p<0.05,{ }^{* *} p<0.01,{ }^{* * *} p<0.001$

growth, invasive capacity and colony formation activity in SOX10 KD cells to a different extent depending on cell lines (Fig. 4c-g). NEDD9 OE exhibited a similar degree of rescue growth and invasive capacity in both $\mathrm{A} 375 \mathrm{M}$ and WM266-4 cells (Fig. 4c-e). In contrast, only WM266-4 showed a complete restoration in the number of colonies formed in SOX10 KD by NEDD9 OE while the only partial rescue was observed in A375M cells (Fig. 4f,g). Importantly, the restoration of oncogenic phenotypes in SOX10 KD + NEDD9 OE cells was not due to an increase in SOX9 expression level which is comparable to that in SOX10 KD cells (Fig. 4a,b). While SOX9 KD partly alleviated the anti-tumorigenic effects of SOX10 KD without altering NEDD9 expression (Fig. 3d), NEDD9 OE was able to further restore the proliferation, invasive and colony formation capacity in SOX10 KD + SOX9 KD cells (Additional file 1). Altogether, these results indicate that NEDD9 can mediate most if not all of the metastatic and tumorigenic functions of SOX10.

\section{High level of SOX9 expression is metastatic and oncogenic}

Although our previous studies in chick embryos demonstrated that overexpression of SOX9 was sufficient to 
induce ectopic Nedd9 expression [22], our data showed that moderate increase of SOX9 expression in SOX10 KD melanoma cells was not able to restore NEDD 9 expression (Fig. 3c,d and Fig. 4a,b), raising the possibility that further elevation of SOX9 expression level might be required for the restoration of NEDD9 expression based on the previous findings that SOX9 acts in a dose-dependent manner $[34,35]$. Indeed, SOX9 OE at the highest titer $(200 \mu \mathrm{L})$ in both SOX10 KD A375M and WM266-4 melanoma cells significantly restored the levels of NEDD9 mRNA and protein expression of both parental and phosphorylated forms compared to SOX10 KD alone (Fig. 4a,b). Consistently, SOX9 OE restored proliferation and colony formation of SOX10 KD cells to a greater extent (Fig. 4c,f,g), but partly rescued on invasion in both SOX10 KD A375M and WM266-4 cells (Fig. 4d,e). These in vitro findings suggest that high level of SOX9 expression is metastatic/tumorigenic in melanoma cells.

\section{SOX9 transactivates NEDD9 expression in a dose- dependent manner}

To further demonstrate the dose-dependent effects of SOX9 on NEDD9 expression, we transduced SOX10 KD A375M and WM266-4 melanoma cells with a gradual increase in titer of lentiviral vector expressing SOX9 $(50 \mu \mathrm{L}$ to $200 \mu \mathrm{L})$ followed by assessment of SOX9, SOX10 and NEDD9 genes and protein expression levels (Fig. 5a,b). The results showed that progressive increase in the amount of lentiviruses expressing SOX9 resulted in a dose-dependent increase in the expression levels of SOX9 and NEDD9 in the SOX10 low environment (Fig. $5 \mathrm{a}, \mathrm{b})$, indicating that $S O X 9 \mathrm{OE}$ is able to restore NEDD9 expression in a dose-dependent manner in SOX10 KD melanoma cells.

To further determine whether SOX10 and/or SOX9 can regulate NEDD9 expression through transactivating its promoter, we performed luciferase reporter assay driven by the NEDD9 promoter $(\sim 1 \mathrm{~kb})$ in both A375M and WM266-4 melanoma cell lines. The results showed that SOX10 OE $(200 \mu \mathrm{L})$ exhibited a greater extent than SOX9 OE $(200 \mu \mathrm{L})$ in the transactivation of the NEDD9 promoter activity in parental cells (Fig. 5c). In agreement with this, chromatin immunoprecipitation (ChIP) assay in A375M cells indicated that SOX10 has a higher binding affinity than SOX9 for a SOX consensus motif (AAACAAA) within the NEDD9 promoter compared to IgG control (Fig. 5d), whereas none of these proteins bound to another DNA fragment without the motif, indicating the specificity of the binding (data not shown). In contrast, SOX10 KD significantly reduced the $N E D D 9$-reporter activity compared to the control, while SOX9 OE restored the reporter activity in a dose-dependent manner in both cell lines (Fig. 5c). These data further confirm that high levels of SOX9 expression were able to induce and restore NEDD9 expression partly through binding and transactivating its promoter in both wild-type and SOX10 low environment, respectively (Fig. 5a-e).

Consistent with the observations that $\mathrm{SOX} 9 \mathrm{OE}$ was able to restore the invasive behavior of SOX10 KD A375M and WM266-4 cells, SOX9 OE was also sufficient to promote the invasiveness of their parental forms compared to vehicle control (Fig. 5f,g). We then examined the impact of SOX9 OE on a panel of matrix metalloproteinases (MMPs) expression which has been implicated in promoting melanoma metastasis through proteolysis of extracellular matrix [36]. qPCR analysis revealed that $M M P 1$, $M M P 7$, and $M M P 23$ expression were upregulated in both cell lines treated with $S O X 9$ OE compared to the vehicle control, whereas MMP8 expression was downregulated. However, only A375 but not WM266-4 cells exhibited a robust elevation of $M M P 3$ expression in response to SOX9 OE (Fig. 5h). These findings are consistent with the roles of MMP1, MMP3, and MMP7 as pro-metastatic factors [37-39], and MMP8 as a negative regulator in melanoma invasiveness [40]. Intriguingly, the high MMP23 expression is associated with poor responses to immunotherapy [41]. Altogether, these results suggest that SOX9 OE not only promotes melanoma invasion through modulation of various $M M P$ genes expression but also may have a role in immunosuppression.

\section{SOX9 overexpression restores metastasis in SOX10 silenced melanoma cells in vivo}

The restoration of metastatic capacity in SOX10 KD cells in vitro by NEDD9 OE and SOX9 OE prompted us to examine whether the similar phenomenon occurs in vivo. Following tail vein injection of A375M cells $\left(1 \times 10^{6}\right)$ in NOD/SCID mice, cells treated with the scramble control displayed lung colonization 2 weeks post-injection, whereas no pulmonary metastases were detected from SOX10 KD and NEDD9 KD cells (Fig. 6a-d). Moreover, SOX9 OE at the highest titer $(200 \mu \mathrm{L})$ exhibited a higher capability than NEDD9 $\mathrm{OE}$ in restoring the metastatic capacity of SOX10 KD cells (Fig. 6a-d). Consistent with the ability of SOX9 OE to restore NEDD9 expression in SOX10 KD cells in vitro, we detected ectopic NEDD9 expression in SOX9 overexpressing cells on the section of lung nodule derived from SOX10 KD + SOX9 OE (Fig. 6 e), indicating cell-autonomous induction of NEDD9 by SOX9 OE. These results indicate that increased levels of SOX9 expression can restore the metastatic capacity of SOX10 KD cells and NEDD9 expression in vivo.

\section{SOXE and NEDD9 govern migration dynamics of melanoma cells}

To evaluate the migratory behavior of melanoma cells transduced with the above 5 different treatments, we performed in vitro time-lapse imaging of wound healing 
A
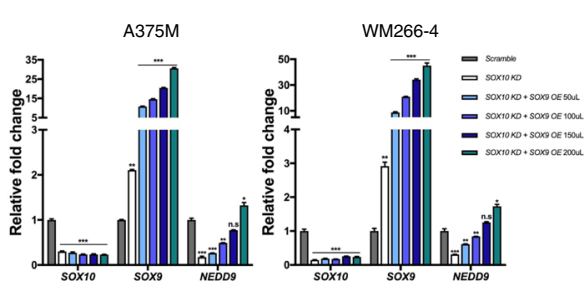

B

C
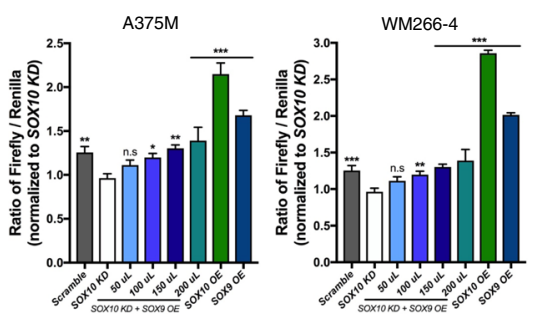

B

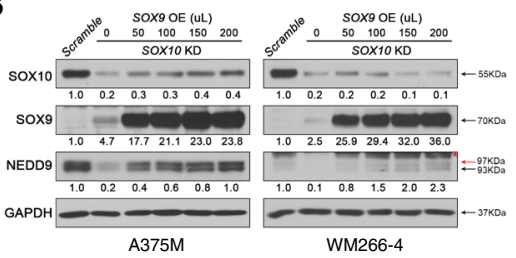

D

WM266-4
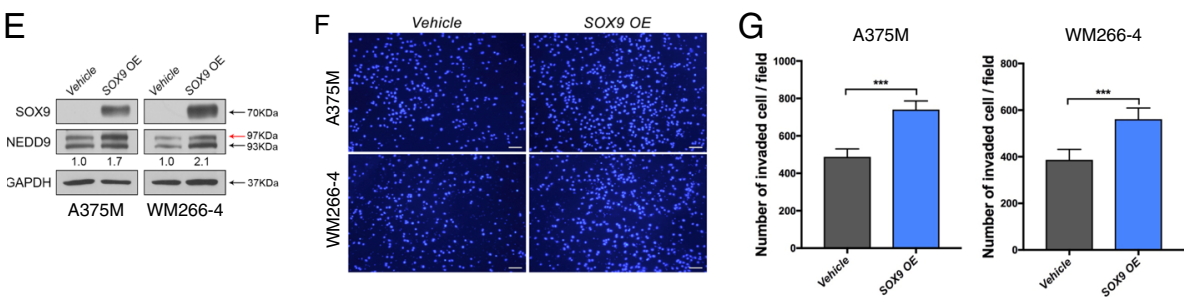

$\mathrm{H}$
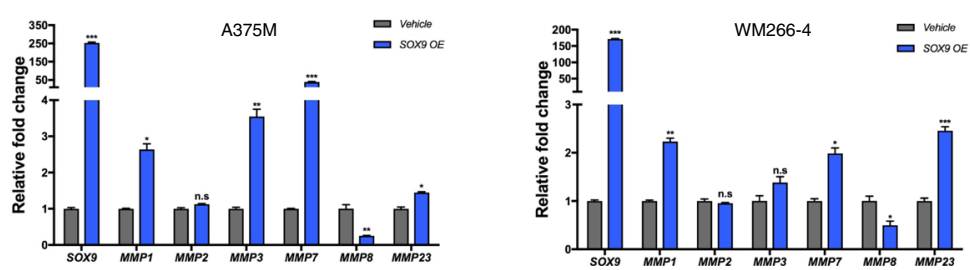

Fig. 5 Overexpression of SOX9 transactivates NEDD9 and induces MMPs expression. a qPCR analysis for the expression levels of SOX10, SOX9, and NEDD9 in A375M and WM266-4 cell lines treated with scramble control, SOX10 KD alone and SOX10 KD together with increasing amount of SOX9 OE lentiviruses. $\mathbf{b}$ Immunoblotting for the indicated antibodies on protein lysates derived from A375M and WM266-4 cells treated with the indicated constructs. The intensity of protein bands in arbitrary units for SOX10, SOX9, and NEDD9 in each melanoma cell line is relative to scramble control which is set to 1 as a reference. The red arrow indicates the phosphorylated form of NEDD9. Asterisk indicates non-specific bands. GAPDH serves as a loading control. c A375M and WM266-4 cells were transfected with a 1 kb-NEDD9 promoter-driven luciferase reporter construct plus renilla for normalization of transfection efficiency together with scramble control, SOX10 KD, SOX10 KD plus increasing amount of SOX9 OE lentiviruses, SOX10 OE, and SOX9 OE. Fold activation of three independent luciferase assays. SOX10 KD is set to 1 as a reference. $\mathbf{d}$ Schematic diagram showing the presence of a SOX binding motif within the $167 \mathrm{bp}$ DNA fragment detected by ChIP-qPCR whereas the 284 bp fragment serves as a negative control. ChIP-qPCR data showing a higher DNA binding capacity by SOX10 than SOX9. Anti-lgG serves as a negative control. e Western blot analysis using the indicated antibodies on protein lysates derived from A375M and WM266-4 cells treated with vehicle control and SOX9 OE. The red arrow indicates the phosphorylated form of NEDD9. $\mathbf{f}$ DAPI images of transwell invasion of melanoma cells treated with the indicated constructs. $\mathbf{g}$ Transwell invasion assay for each cell line treated with the vehicle alone and SOX9 OE lentivirus. Scale bars: $100 \mu \mathrm{M}$. $\mathbf{h}$ mRNA expression of SOX9 and members of MMP family were quantified by qRT-PCR in A375M and WM266-4 cells treated with the vehicle alone and SOX9 OE. Error bars represent mean \pm SD of three independent experiments. n.s, non-significant; ${ }^{*} p<0.05,{ }^{* *} p<0.01,{ }^{* * *} p<0.001$

assay for A375 cells, which were transfected with Lifeact-mCherry to label actin cytoskeleton for monitoring real-time morphological change (Fig. 7a). In the $7.5 \mathrm{~h}$ period of live cell imaging, we found that cells expressing scrambled shRNA migrated to the wound at an average speed of $15 \mu \mathrm{M} / \mathrm{h}$ and exhibited a mesenchymal mode of migration with membrane protrusions at the cell front (Fig. 7a,b and Additional file 2: Movie S1). Conversely, SOX10 KD cells were in round shape and acquired amoeboid migration
(Additional file 3: Movie S2), while NEDD9 KD cells tended to migrate in a cluster with elongated morphology (Additional file 4: Movie S3). Both treatments significantly reduced the speed of migration compared to the control (Fig. 7b). However, cells expressing SOX10 KD + SOX9 OE or SOX10 KD + NEDD9 OE restored the mesenchymal morphology with migration speed similar to the control (Fig. 7a,b and Additional file 5: Movie S4 and Additional file 6: Movie S5). These data demonstrate that SOXE and NEDD9 are 
A
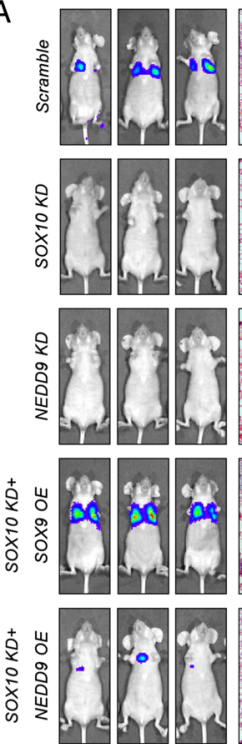

$\mathrm{E}$

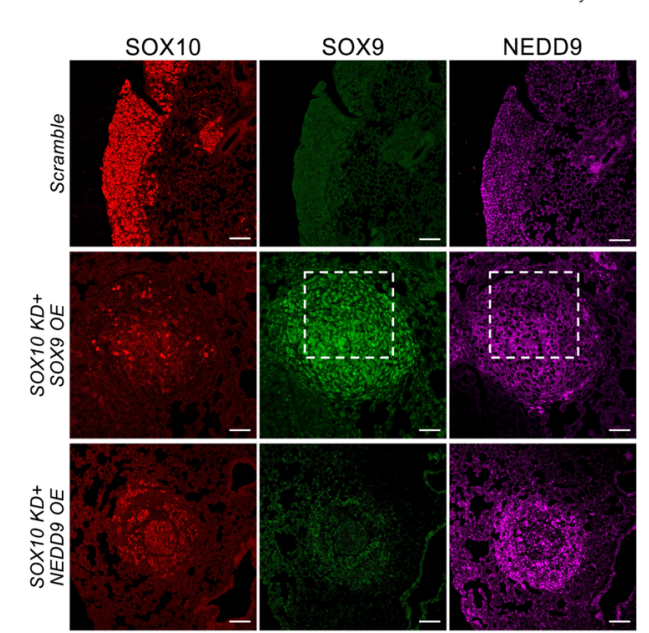

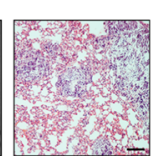
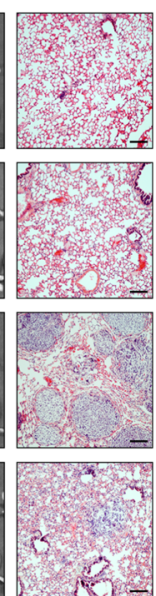

B

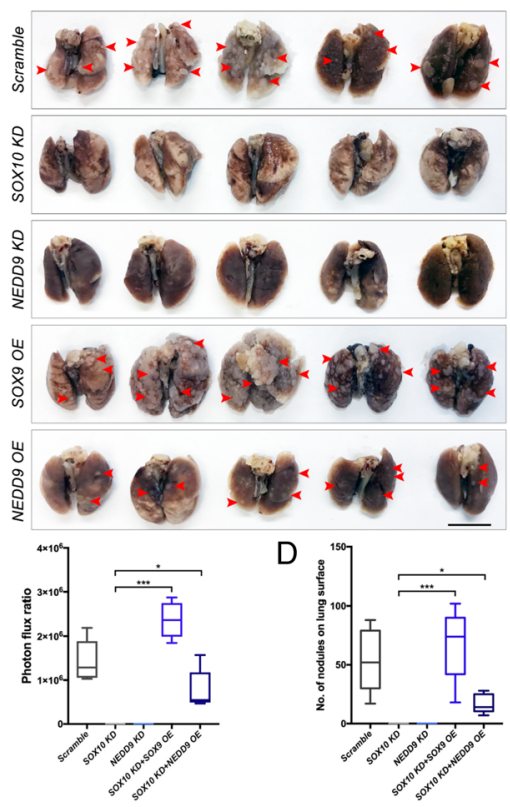

\section{C}

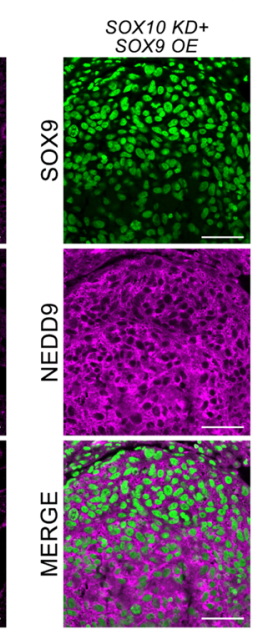

Fig. 6 Overexpression of SOX9 restores lung metastasis in SOX10 KD melanoma cells. a A375M cells treated with the indicated constructs were injected via the tail vein into NOD/SCID mice ( $n=5$ per treatment) and the bioluminescence images were taken 2 weeks after injection. H\&E staining was used to detect for the presence of tumor tissues in lungs of NOD/SCID mice. Scale bars: $200 \mu \mathrm{M}$. $\mathbf{b}$ Gross pictures of lungs from xenografted NOD/SCID mice. The red arrowheads indicate the macroscopic lesions. Scale bar: $1.0 \mathrm{~cm}$. c Bioluminescence intensity was measured and plotted. $\mathbf{d}$ Quantification of the number of nodules formed on the lung surface of NOD/SCID mice injected with the indicated constructs. e Immunofluorescence detection for SOX10, SOX9, and NEDD9 on the cross-section of lung nodules derived from A375M cells treated with scramble control, SOX10 KD + SOX9 OE and SOX10 KD + NEDD9 OE. Magnification of the boxed regions with an overlapping expression of SOX9 and NEDD9. Scale bar: $100 \mu \mathrm{M}$. Error bars represent mean \pm SD of three independent experiments. ${ }^{*} p<0.05, p^{* * *}<0.001$

crucial for promoting migration dynamics of melanoma cells.

SOXE directs mesenchymal-type of melanoma migration through regulation of focal adhesion dynamics and rho GTPases signaling

Previous studies showed that NEDD9 exhibits both positive and negative roles in regulating focal adhesion dynamics and cell motility depending on the cellular context [42, 43] and also contributes to the mesenchymal-type of melanoma migration via modulation of small Rho GTPase activity [29]. Thus, we anticipated that SOX10 or high level of SOX9 regulates NEDD9 expression to promote melanoma migration through alteration of focal adhesion dynamics and RHO signaling activity. To address this issue, we first performed immunofluorescence in A375M cells with five different treatments for vinculin which is one of the key focal adhesion proteins [44] together with phalloidin to mark stress fibers for monitoring cell shape change 

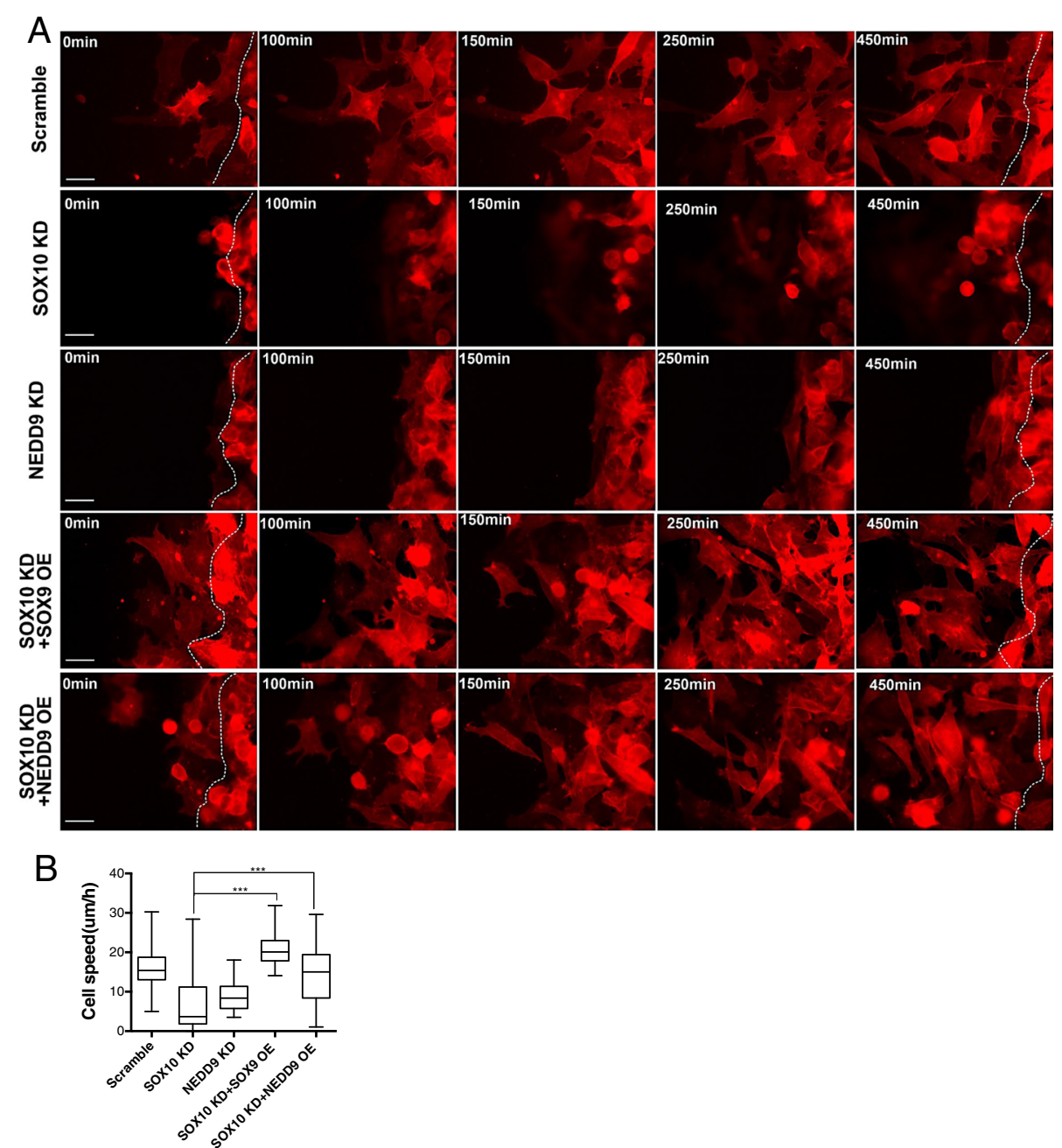

Fig. 7 SOXE and NEDD9 direct migration dynamics of melanoma cells. a Time-lapse imaging showing the migration dynamics and morphology of A375 cells treated with the indicated constructs and Lifeact-mCherry to mark actin cytoskeleton. White dotted lines indicate the border of the wound. Scale bars: $50 \mu \mathrm{M}$. b Quantification of the total speed of A375M cells treated with scramble control $(n=57)$, SOX10 KD $(n=52)$, NEDD9 ( $n=51)$, SOX10 KD + SOX9 OE $(n=67)$ and SOX10 KD + NEDD9 OE $(n=38)$. Error bars represent mean \pm SD of three independent experiments. $p^{* * *}<0.001$

(Fig. 8a). The dynamic exchange rate (assembly and disassembly) of vinculin will be assessed by quantification of its numbers, area covered and size at the focal adhesion site to indicate positive or negative regulation of cell migration (Fig. 8b-d). The results showed that SOX10 $\mathrm{KD}$ and NEDD9 KD melanoma cells exhibited an increased number of vinculin ${ }^{+}$focal adhesions together with larger average area and size per cell compared to the control that could result in enhanced focal adhesion contact with the substratum in vitro and reduced cell motility (Fig. 4d,e, Fig. 7a,b and Fig. 8b-d). In contrast, SOX9 OE and NEDD9 OE restored the number; area and size of focal adhesion in SOX10 KD A375M cells similar to that observed in the control, and rescued their migratory capacity accordingly (Fig. 4d,e, Fig. 7a,b and Fig. 8b-d). It has been well established that RHOA activation is associated with enhanced focal adhesion formation, and the mutual antagonism between RHOA and $\mathrm{RAC} 1$ determines cell shape and mode of migration: RHOA-Rho-associated kinase (ROCK) signaling is associated with the amoeboid morphology and RAC1 is associated with the mesenchymal phenotype $[30,45,46]$. In agreement with this, we detected the elevation of RHOA and reduction of RAC1 activities in both SOX10 $\mathrm{KD}$ and NEDD9 KD cells which are predominantly amoeboid and elongated with relatively less sheet-like protrusions consistent with previous observations in live cell imaging studies (Fig. 7a and Fig. 8a,e,f). This is in contrast to the scramble control, which exhibits a relatively high RAC1 than RHOA activity that is associated with mesenchymal morphology (Fig. 7a and Fig. 8a,e). Conversely, SOX9 OE and NEDD9 OE led to a reduction of RHOA and upregulation RAC1 activities in SOX10 KD cells accompanied by acquisition of mesenchymal 


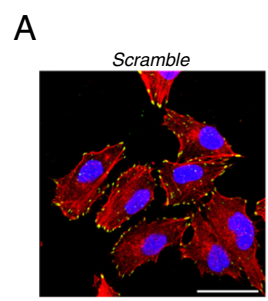

B

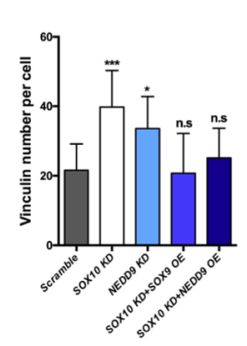

E

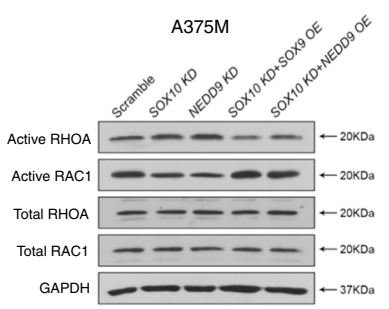

G

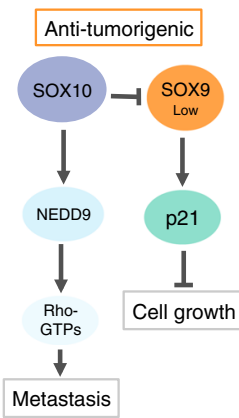

SOX10 KD+SOX9 OE

OX10 KD+NEDD9 OE

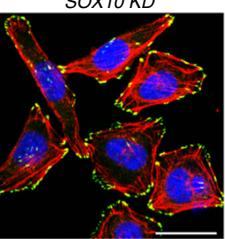

$F$

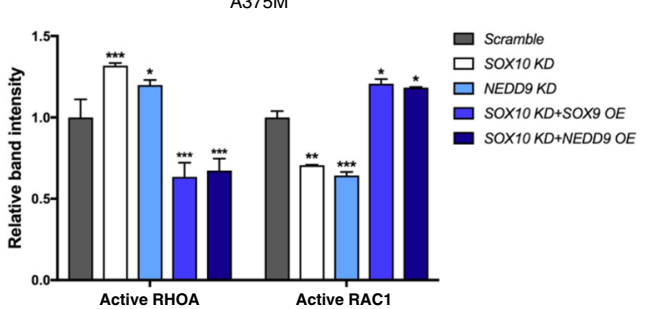

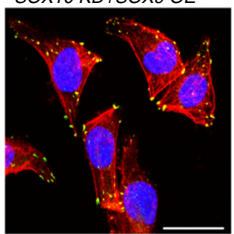

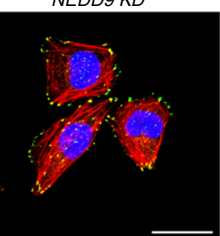

D
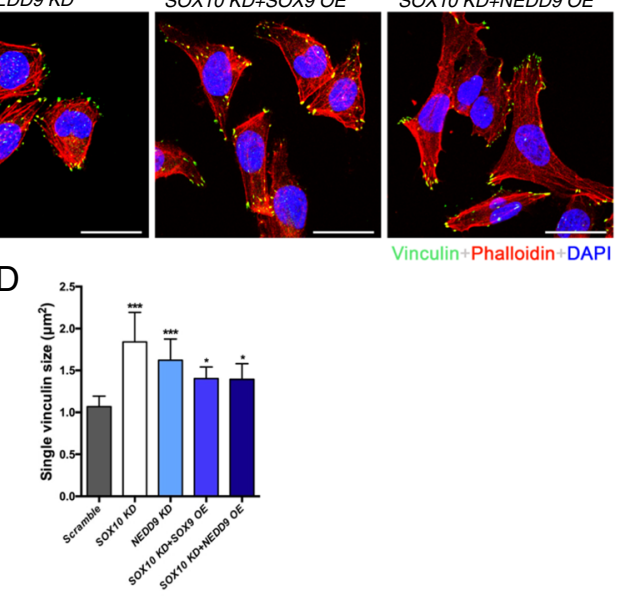

C

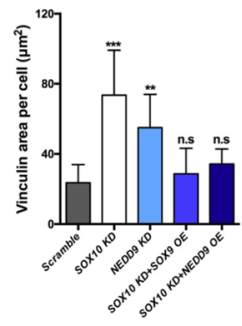

Vinculin+Phalloidin+DAPI

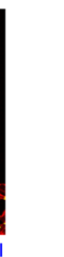


$[17,18,20,48]$. Here we demonstrate that SOX10 is expressed in melanocytic nevus, primary cutaneous, and invasive melanomas where SOX9 exhibits unique but overlapping expression with SOX10. Both SOX9 and SOX10 are co-expressed with pro-metastasis factor NEDD9 to different extents and levels. In agreement with this, SOX10 and/or high SOX9 are required for NEDD9 expression, which is partly responsible for their metastatic properties both in vitro and in vivo. Thus, the levels of the upregulated SOX9 expression in SOX10 KD melanoma cell lines are similar to the low mRNA levels of SOX9 detected in cutaneous melanoma specimens. These levels of SOX9 expression are able to trigger p21 but not sufficient to induce NEDD9 expression, resulting in suppression of tumor growth and metastasis. This explains why low levels of SOX9 expression are negatively correlated with NEDD9 in most primary melanoma specimens. In contrast, further elevation of SOX9 dosage corresponding to high SOX9 in metastatic melanoma specimens lead to opposite effects on p21 and NEDD9 expression with enhanced tumor growth and metastasis as well as induction of MMPs expression. Lastly, SOX10 or high SOX9 regulates focal adhesion turnover and Rho GTPase signaling to promote mesenchymal migration of melanoma cells. Altogether, our studies provide a molecular explanation to reconcile the previous discrepancies that anti-metastatic role of SOX9 is conferred by its sub-optimal level of expression while a high level of SOX9 is pro-metastatic in a heterogeneous population of melanoma (Fig. 8g).

SOX9 has been shown to play an oncogenic role in the formation and growth of tumors in the prostate, the CNS, skin, pancreas, liver, and esophagus [49-53]. However, the previous study by Passeron et al. demonstrated that overexpression of SOX9 in A375 cells inhibited proliferation and tumor growth in xenografts [18]. Similarly, another study by Cheng et al. also showed cell cycle arrest when SOX9 was overexpressed in proliferative melanoma cell lines M010817 and M980513 [20]. Our findings suggest that upregulated SOX9 expression levels in SOX10 KD is probably similar to the levels of SOX9 overexpression from these two independent studies and that is sufficient to activate p21 expression for slow growth rate of cells. In addition, we further revealed that elevation of SOX9 expression level resulted in downregulation of p21 and restoration of melanoma proliferation and growth. These data clearly indicate that distinct levels of SOX9 expression impinge on the differential regulation of p21 expression. This dose-dependent effect of SOX9 is also implicated in colorectal cancer model in which a critical dose of SOX9 activity is essential for a maximum rate of proliferation while expression levels higher or lower than this dose would result in the reduction of cell growth [54]. In contrast, we found that overexpression of SOX9 did not have obvious effect on
SOX10 expression that differs from a previous report which showed a pronounced downregulation of SOX10 protein by SOX9 overexpression in A375 and M010817 cells [17]. Although the reason for these discrepancies is unclear, it might be caused by using different types of vector (lentiviral vs plasmid) for overexpression that could lead to differential effects of SOX9 on SOX10 expression.

Cheng et al. further showed that overexpression of SOX9 using the same proliferative melanoma cell lines increased their invasiveness to the lungs after intravenous injection [20]. These results are in agreement with our observations that SOX9 overexpression promoted melanoma metastasis in SOX10 KD both in vitro and in vivo. Furthermore, our immunohistochemistry showed the detection of SOX9 mRNA and protein exclusively in the metastatic melanomas, that is in accord with a previous study in which high SOX9 is associated with lower survival rates of patients with advanced melanoma [20]. The distinct patterns of SOX9 and SOX10 expression in patient specimens probably reflect the heterogeneity of melanoma population harboring different genetic and epigenetic signatures since SOX9 expression could be regulated by DNA methylation [20] and displays antagonistic relationship with SOX10 [17]. Based on our findings together with others, we propose that SOX10 inhibits SOX9 and/or SOX9 promoter is methylated that maintain SOX9 expression at low or sub-optimal level in primary melanoma. As melanoma acquire invasiveness, the promoter of SOX9 becomes hypomethylated probably through downregulation of DNA methyltransferase [20] that could partly contribute to its high level of expression in a subset of SOX10 negative metastatic melanoma.

Although both SOX9 and SOX10 exhibit differential expression patterns in melanomas, whether they share the same or distinct transcriptional targets in mediating the oncogenic events is not known. A previous report revealed that SOX10 transactivates MIA expression through its promoter to induce invasive capacity of melanoma cells. Intriguingly, SOX9 activates $M I A$ in chondrocytes during mouse chondrogenesis [55]. Likewise, we recently identified NEDD9 as a direct transcriptional target of SOX9 in mediating avian neural crest delamination [22], whereas the present study suggests that both SOX9 and SOX10 are able to regulate NEDD9 expression partly through transactivating its promoter region. These results suggest that SOX9-regulated developmental genes are being adopted and regulated by SOX10 in controlling the invasive behavior of melanoma. Whether the switching of transcriptional targets occurs only between closely related transcription factors remains to be determined. This could be due to evolutionary change of a few nucleotides within the human gene promoter 
different from its mouse and chick counterparts that might alter the binding affinity and transactivation capacity between the two closely related transcription factors. In agreement with this notion, our reporter and ChIP assays suggest that the human NEDD9 promoter sequence favors the binding and transactivation capacity by SOX10 whereas the high level of SOX9 expression is required for efficient activation of NEDD9 expression in both parental and SOX10 KD melanoma cells. On the other hand, the low level of SOX9 contributes to the p21 activation whereas the high level inhibits its expression. The molecular mechanism underlying the dichotomous role of SOX9 in gene regulation is not known. Given the importance of cofactors in conferring tissue-specific action of SOX9 [56], it is conceivable that distinct SOX9 expression levels may associate with different cofactors to orchestrate differential regulation of target genes and the subsequent impact on melanoma growth and invasion. The identity of these cofactors remains to be discovered by a mass spectrometry-based proteomic method.

Although NEDD9 has been shown to be involved in promoting melanoma metastases [28, 57], the present data showed that it is rather less efficient in restoring pulmonary metastases of SOX10 KD cells in vivo compared to in vitro, probably due to complex in vivo environment that might alter cellular states and responses. In contrast, elevation of SOX9 expression is highly effective in restoring melanoma invasiveness in SOX10 KD cells. This is likely because SOX9 acting upstream of NEDD9 can regulate multiple downstream targets as previously demonstrated by RNAseq analysis, which revealed a few novel candidates that could potentially drive the invasive melanoma phenotype [20]. Indeed, our data showed that high SOX9 not only activates NEDD9 expression to promote mesenchymal migration of melanoma cells through regulation of Rho GTPase activity but also their invasiveness by modulating the expression of various MMPs that mediate extracellular matrix degradation as well as confer immunosuppressive response. Altogether, our findings demonstrate that distinct transcriptional targets of SOX9 at different levels of expression confer melanomas with various cellular properties. It will be worth to perform RNAseq in melanoma cells expressing various levels of SOX9 expression in order to unravel the sets of downstream target genes responsible for the anti-metastatic and the pro-metastatic effects. The outcome of this study would shed new insight into the dosage-dependent transcriptional regulation of SOX9 and also uncover novel druggable targets for the treatment of this devastating disease.

\section{Conclusions}

In conclusion, our findings unravel NEDD9 as a common transcriptional target for SOX10 or high SOX9 to partly mediate their oncogenic features in melanoma, and most importantly reconcile previous discrepancies that low or sub-optimal level of SOX9 expression dictates its anti-metastatic properties whereas high SOX9 is metastatic in a heterogeneous population of melanoma.

\section{Additional file}

\begin{abstract}
Additional file 1: Overexpression of NEDD9 restores the oncogenic properties of SOX10 KD + SOX9 KD melanoma cells. (A) AlamarBlue assay for each cell line treated with the indicated constructs. (B) Representative images of crystal violet stained A375M and WM266-4 clones subjected to different treatments. (C) Quantification of the number of A375M and WM266-4 colonies treated with the indicated constructs. (D) Quantification of the number of invaded cells treated with the indicated constructs. (E) DAPI images of transwell invasion of melanoma cells treated with the indicated constructs. Error bars represent \pm SD of three independent experiments. ${ }^{*} p<0.05,{ }^{* *} p<0.01$, ${ }^{* *} p<0.001$. (PDF $619 \mathrm{~kb}$ )

Additional file 2: Melanoma cells transduced with scrambled shRNA exhibit mesenchymal-type of movement with membrane protrusions on the front and small-finger like projections in the periphery. This movie file contains 450 min imaging sequences with 5 min intervals between frames, corresponding to the images shown in Fig. 7a. (MOV 10253 kb)
\end{abstract}

Additional file 3: Melanoma cells transduced with SOX10 KD are round in shape and exhibit amoeboid movement. This movie file contains 450 min imaging sequences with 5 min intervals between frames, corresponding to the images shown in Fig. 7a. (MOV 4770 kb)

Additional file 4: Melanoma cells transduced with NEDD9 KO exhibit elongated morphology and migrate in a cluster. This movie file contains 450 min imaging sequences with 5 min intervals between frames, corresponding to the images shown in Fig. 7a. (MOV 7524 kb)

Additional file 5: Melanoma cells transduced with SOX10 KD + SOX9 OE restore mesenchymal-type of movement with membrane protrusions on the front towards the direction of motion. This movie file contains 450 min imaging sequences with 5 min intervals between frames, corresponding to the images shown in Fig. 7a. (MOV 10444 kb)

Additional file 6: Melanoma cells transduced with SOX10 KD + NEDD9 OE display switching from amoeboid to mesenchymal migration. This movie file contains 450 min imaging sequences with 5 min intervals between frames, corresponding to the images shown in Fig. 7a. (MOV 10208 kb)

\section{Abbreviations}

CAS: a member of the Crk-associated substrate; KD: knockdown; MMP: matrix metalloproteinase; NC: neural crest; NEDD9: Neural precursor expressed, developmentally down-regulated 9; OE: overexpression; SOXE: (Sry (Sex determining gene)-HMG box) E)

\section{Acknowledgements}

The authors thank the doctors and the patients who participated in our study as well as Dr. Jason Wong from the School of Biomedical Sciences in HKU for his assistance and advice on bioinformatic analysis. Animals and live cell imaging were acquired using equipment maintained by the University of Hong Kong Li Ka Shing Faculty of Medicine Faculty Core Facility. We thank the technical staff in the Faculty Core Facility for their assistance and advice on live cell and animal imaging. This work was supported by grants from the Research Grants Council and University Grants Council of Hong Kong (GRF_17110715, GRF_17123016, GRF_17114817) as well as Small Project Funding (201409176166) and Seed Fund for Basic Research (201711159209) from the University of Hong Kong. 


\section{Funding}

This work was supported by grants from the Research Grants Council and University Grants Council of Hong Kong (GRF_17110715, GRF_17123016, GRF_17114817) as well as Small Project Funding (201409176166) and Seed Fund for Basic Research (201711159209) from the University of Hong Kong.

\section{Availability of data and materials}

All data generated or analyzed during this study are included in the published article and its supplementary information files.

\section{Authors' contributions}

Conceived and designed the experiments: $X T Y, R L, H L L$ and $M C$. Performed the experiments: $X T Y, R L, M P L C$ and OY M. Analyze the data: $X T Y, R L, J A I$ $L, H L L$ and $M C$. Melanoma cell lines: $X Y$ G. Patient specimens: $C X L$ and $X L L$. Wrote the paper: $X T Y, R L, H L L$ and $M C$. All authors read and approved the final manuscript.

\section{Ethics approval and consent to participate}

The Committee on the Use of Live Animals in Teaching and Research in the University of Hong Kong (CULATR no: 4005-16) approved all the experimental procedure involving animal experimentation.

Surgically procured tumor samples from patients with nevus, primary cutaneous and metastatic melanomas were obtained in the Department of Anesthesiology, Zhejiang Cancer Hospital and Department of Pediatric Surgery, the Second Hospital of Hebei Medical University with informed patients' consent for research purposes.

\section{Consent for publication}

Not applicable.

\section{Competing interests}

The authors declare that they have no competing interest.

\section{Publisher's Note}

Springer Nature remains neutral with regard to jurisdictional claims in published maps and institutional affiliations.

\section{Author details}

${ }^{1}$ School of Biomedical Sciences, Li Ka Shing Faculty of Medicine, The University of Hong Kong, 21 Sassoon Road, Hong Kong, China. ${ }^{2}$ Department of Anesthesiology, Zhejiang Cancer Hospital, Hangzhou, Zhejiang, China. ${ }^{3}$ Department of Pediatric Surgery, Second Hospital of Hebei Medical University, Shijiazhuang, Hebei, China. ${ }^{4}$ Department of Biology, Faculty of Science, Hong Kong Baptist University, Hong Kong, China. ${ }^{5}$ Department of Clinical Oncology, Li Ka Shing Faculty of Medicine, The University of Hong Kong, Hong Kong, China.

Received: 18 September 2018 Accepted: 6 December 2018 Published online: 14 January 2019

\section{References}

1. Siegel RL, Miller KD, Jemal A. Cancer statistics, 2015. CA Cancer J Clin. 2015; 65(1):5-29.

2. Hill VK, Gartner JJ, Samuels Y, Goldstein AM. The genetics of melanoma: recent advances. Annu Rev Genomics Hum Genet. 2013;14:257-79.

3. Queirolo P, Picasso V, Spagnolo F. Combined BRAF and MEK inhibition for the treatment of BRAF-mutated metastatic melanoma. Cancer Treat Rev. 2015;41(6):519-26.

4. Spagnolo F, Ghiorzo P, Orgiano L, Pastorino L, Picasso V, Tornari E, et al. BRAF-mutant melanoma: treatment approaches, resistance mechanisms, and diagnostic strategies. Onco Targets Ther. 2015;8:157-68.

5. Ennen M, Keime C, Kobi D, Mengus G, Lipsker D, Thibault-Carpentier C, et al. Single-cell gene expression signatures reveal melanoma cell heterogeneity. Oncogene. 2015;34(25):3251-63.

6. Cheung M, Briscoe J. Neural crest development is regulated by the transcription factor Sox9. Development. 2003;130(23):5681-93.

7. Southard-Smith EM, Kos L, Pavan WJ. Sox10 mutation disrupts neural crest development in Dom Hirschsprung mouse model. Nat Genet. 1998;18(1): $60-4$.

8. Liu JA, Wu MH, Yan CH, Chau BK, So H, Ng A, et al. Phosphorylation of Sox9 is required for neural crest delamination and is regulated downstream of
BMP and canonical Wht signaling. Proc Natl Acad Sci U S A. 2013;110(8): 2882-7.

9. Liu JA, Cheung M. Neural crest stem cells and their potential therapeutic applications. Dev. Biol. 2016;419(2):199-216.

10. Cheung M, Chaboissier MC, Mynett A, Hirst E, Schedl A, Briscoe J. The transcriptional control of trunk neural crest induction, survival, and delamination. Dev Cell. 2005;8(2):179-92.

11. McKeown SJ, Lee VM, Bronner-Fraser M, Newgreen DF, Farlie PG. Sox10 overexpression induces neural crest-like cells from all dorsoventral levels of the neural tube but inhibits differentiation. Dev Dyn. 2005;233(2):430-44.

12. Harris ML, Buac K, Shakhova O, Hakami RM, Wegner M, Sommer L, et al. A dual role for SOX10 in the maintenance of the postnatal melanocyte lineage and the differentiation of melanocyte stem cell progenitors. PLoS Genet. 2013;9(7):e1003644.

13. Harris ML, Baxter LL, Loftus SK, Pavan WJ. Sox proteins in melanocyte development and melanoma. Pigment Cell Melanoma Res. 2010;23(4): 496-513.

14. Potterf SB, Mollaaghababa R, Hou L, Southard-Smith EM, Hornyak TJ, Arnheiter $\mathrm{H}$, et al. Analysis of SOX10 function in neural crest-derived melanocyte development: SOX10-dependent transcriptional control of dopachrome tautomerase. Dev Biol. 2001;237(2):245-57.

15. Kelsh RN. Sorting out Sox10 functions in neural crest development. BioEssays. 2006;28(8):788-98.

16. Cronin JC, Watkins-Chow DE, Incao A, Hasskamp JH, Schonewolf N, Aoude LG, et al. SOX10 ablation arrests cell cycle, induces senescence, and suppresses melanomagenesis. Cancer Res. 2013;73(18):5709-18.

17. Shakhova O, Cheng P, Mishra PJ, Zingg D, Schaefer SM, Debbache J, et al. Antagonistic cross-regulation between Sox9 and Sox 10 controls an antitumorigenic program in melanoma. PLoS Genet. 2015;11(1):e1004877.

18. Passeron T, Valencia JC, Namiki T, Vieira WD, Passeron H, Miyamura $Y$, et al. Upregulation of SOX9 inhibits the growth of human and mouse melanomas and restores their sensitivity to retinoic acid. J Clin Invest. 2009; 119(4):954-63.

19. Graf SA, Busch C, Bosserhoff AK, Besch R, Berking C. SOX10 promotes melanoma cell invasion by regulating melanoma inhibitory activity. J Invest Dermatol. 2014;134(8):2212-20.

20. Cheng PF, Shakhova O, Widmer DS, Eichhoff OM, Zingg D, Frommel SC, et al. Methylation-dependent SOX9 expression mediates invasion in human melanoma cells and is a negative prognostic factor in advanced melanoma. Genome Biol. 2015;16:42.

21. Flammiger A, Besch R, Cook AL, Maier T, Sturm RA, Berking C. SOX9 and SOX10 but not BRN2 are required for nestin expression in human melanoma cells. J Invest Dermatol. 2009;129(4):945-53.

22. Liu JA, Rao Y, Cheung MPL, Hui MN, Wu MH, Chan LK, et al. Asymmetric localization of DLC1 defines avian trunk neural crest polarity for directional delamination and migration. Nat Commun. 2017;8(1):1185.

23. Aquino JB, Lallemend F, Marmigere F, Adameyko II, Golemis EA, Ernfors P. The retinoic acid inducible Cas-family signaling protein Nedd9 regulates neural crest cell migration by modulating adhesion and actin dynamics. Neuroscience. 2009;162(4):1106-19.

24. Li Y, Bavarva JH, Wang Z, Guo J, Qian C, Thibodeau SN, et al. HEF1, a novel target of Wnt signaling, promotes colonic cell migration and cancer progression. Oncogene. 2011;30(23):2633-43.

25. Minn AJ, Gupta GP, Siegel PM, Bos PD, Shu W, Giri DD, et al. Genes that mediate breast cancer metastasis to lung. Nature. 2005;436(7050):518-24.

26. Natarajan M, Stewart JE, Golemis EA, Pugacheva EN, Alexandropoulos K, Cox BD, et al. HEF1 is a necessary and specific downstream effector of FAK that promotes the migration of glioblastoma cells. Oncogene. 2006;25(12):1721-32.

27. Kim SH, Xia D, Kim SW, Holla V, Menter DG, Dubois RN. Human enhancer of filamentation 1 is a mediator of hypoxia-inducible factor-1alpha-mediated migration in colorectal carcinoma cells. Cancer Res. 2010;70(10):4054-63.

28. Kim M, Gans JD, Nogueira C, Wang A, Paik JH, Feng B, et al. Comparative oncogenomics identifies NEDD9 as a melanoma metastasis gene. Cell. 2006; 125(7):1269-81.

29. Sanz-Moreno V, Gadea G, Ahn J, Paterson H, Marra P, Pinner S, et al. Rac activation and inactivation control plasticity of tumor cell movement. Cell. 2008;135(3):510-23.

30. Ahn J, Sanz-Moreno V, Marshall CJ. The metastasis gene NEDD9 product acts through integrin beta3 and Src to promote mesenchymal motility and inhibit amoeboid motility. J Cell Sci. 2012;125(Pt 7):1814-26. 
31. Domingues MJ, Rambow F, Job B, Papon L, Liu W, Larue L, et al. betacatenin inhibitor ICAT modulates the invasive motility of melanoma cells. Cancer Res. 2014;74(7):1983-95.

32. Danga ME, Yaar $R$, Bhawan J. Melan-A positive dermal cells in malignant melanoma in situ. J Cutan Pathol. 2015;42(6):388-93.

33. Guo R, Franco-Palacios M, Russell M, Goddard L, Hassell L, Gillies E, et al. Micropthalmia transcription factor (MITF) as a diagnostic marker for metastatic melanomas negative for other melanoma markers. Int J Clin Exp Pathol. 2013;6(8):1658-64.

34. Wagner T, Wirth J, Meyer J, Zabel B, Held M, Zimmer J, et al. Autosomal sex reversal and campomelic dysplasia are caused by mutations in and around the SRY-related gene SOX9. Cell. 1994;79(6):1111-20.

35. Formeister EJ, Sionas AL, Lorance DK, Barkley CL, Lee GH, Magness ST. Distinct SOX9 levels differentially mark stem/progenitor populations and enteroendocrine cells of the small intestine epithelium. Am J Physiol Gastrointest Liver Physiol. 2009;296(5):G1108-18.

36. Hofmann UB, Westphal JR, Van Muijen GN, Ruiter DJ. Matrix metalloproteinases in human melanoma. J Invest Dermatol. 2000;115(3): 337-44.

37. Blackburn JS, Liu I, Coon Cl, Brinckerhoff CE. A matrix metalloproteinase-1/ protease activated receptor-1 signaling axis promotes melanoma invasion and metastasis. Oncogene. 2009;28(48):4237-48.

38. Nikkola J, Vihinen P, Vlaykova T, Hahka-Kemppinen M, Kahari VM, Pyrhonen S. High expression levels of collagenase- 1 and stromelysin-1 correlate with shorter disease-free survival in human metastatic melanoma. Int J Cancer. 2002;97(4):432-8.

39. Felli N, Felicetti F, Lustri AM, Errico MC, Bottero L, Cannistraci A, et al. miR$126 \& 126^{*}$ restored expressions play a tumor suppressor role by directly regulating ADAM9 and MMP7 in melanoma. PLoS One. 2013;8(2):e56824

40. Gutierrez-Fernandez A, Fueyo A, Folgueras AR, Garabaya C, Pennington CJ, Pilgrim S, et al. Matrix metalloproteinase-8 functions as a metastasis suppressor through modulation of tumor cell adhesion and invasion. Cancer Res. 2008;68(8):2755-63.

41. Moogk D, da Silva IP, Ma MW, Friedman EB, de Miera EV, Darvishian F, et al. Melanoma expression of matrix metalloproteinase-23 is associated with blunted tumor immunity and poor responses to immunotherapy. J Transl Med. 2014;12:342.

42. Zhong J, Baquiran JB, Bonakdar N, Lees J, Ching YW, Pugacheva E, et al. NEDD9 stabilizes focal adhesions, increases binding to the extra-cellular matrix and differentially effects 2D versus 3D cell migration. PLoS One. 2012; 7(4):e35058.

43. Baquiran JB, Bradbury P, O'Neill GM. Tyrosine Y189 in the substrate domain of the adhesion docking protein NEDD9 is conserved with p130Cas Y253 and regulates NEDD9-mediated migration and focal adhesion dynamics. PLoS One. 2013;8(7):e69304.

44. Wozniak MA, Modzelewska K, Kwong L, Keely PJ. Focal adhesion regulation of cell behavior. Biochim Biophys Acta. 2004;1692(2-3):103-19.

45. Lim Y, Lim ST, Tomar A, Gardel M, Bernard-Trifilo JA, Chen XL, et al. PyK2 and FAK connections to p190Rho guanine nucleotide exchange factor regulate RhoA activity, focal adhesion formation, and cell motility. J Cell Biol. 2008;180(1):187-203.

46. Chauhan BK, Lou M, Zheng Y, Lang RA. Balanced Rac1 and RhoA activities regulate cell shape and drive invagination morphogenesis in epithelia. Proc Natl Acad Sci U S A. 2011;108(45):18289-94.

47. Shakhova O, Zingg D, Schaefer SM, Hari L, Civenni G, Blunschi J, et al. Sox10 promotes the formation and maintenance of giant congenital naevi and melanoma. Nat Cell Biol. 2012;14(8):882-90.

48. Ashkenazi S, Ortenberg R, Besser M, Schachter J, Markel G. SOX9 indirectly regulates CEACAM1 expression and immune resistance in melanoma cells. Oncotarget. 2016;7(21):30166-77.

49. Ma F, Ye H, He HH, Gerrin SJ, Chen S, Tanenbaum BA, et al. SOX9 drives WNT pathway activation in prostate cancer. J Clin Invest. 2016;126(5):1745-58.

50. Hiraoka K, Hayashi T, Kaneko R, Nasu-Nishimura Y, Koyama-Nasu R, Kawasaki $Y$, et al. SOX9-mediated upregulation of LGR5 is important for glioblastoma tumorigenicity. Biochem Biophys Res Commun. 2015;460(2):216-21.

51. Larsimont JC, Youssef KK, Sanchez-Danes A, Sukumaran V, Defrance M, Delatte B, et al. Sox9 controls self-renewal of oncogene targeted cells and links tumor initiation and invasion. Cell Stem Cell. 2015;17(1):60-73.

52. Zhou H, Qin Y, Ji S, Ling J, Fu J, Zhuang Z, et al. SOX9 activity is induced by oncogenic Kras to affect MDC1 and MCMs expression in pancreatic cancer. Oncogene. 2018;37(7):912-23.
53. Hong $Y$, Chen W, Du X, Ning $H$, Chen $H$, Shi R, et al. Upregulation of sexdetermining region Y-box 9 (SOX9) promotes cell proliferation and tumorigenicity in esophageal squamous cell carcinoma. Oncotarget. 2015; 6(31):31241-54.

54. Prevostel C, Rammah-Bouazza C, Trauchessec H, Canterel-Thouennon L, Busson $M$, Ychou $M$, et al. SOX9 is an atypical intestinal tumor suppressor controlling the oncogenic Wnt/ss-catenin signaling. Oncotarget. 2016;7(50): 82228-43.

55. Xie WF, Zhang X, Sakano S, Lefebvre V, Sandell L. Trans-activation of the mouse cartilage-derived retinoic acid-sensitive protein gene by Sox9. J Bone Miner Res. 1999:14(5):757-63.

56. Jo A, Denduluri S, Zhang B, Wang Z, Yin L, Yan Z, et al. The versatile functions of Sox9 in development, stem cells, and human diseases. Genes Dis. 2014;1(2):149-61.

57. Rambow F, Bechadergue A, Luciani F, Gros G, Domingues M, Bonaventure J, et al. Regulation of melanoma progression through the TCF4/miR-125b/ NEDD9 Cascade. J Invest Dermatol. 2016;136(6):1229-37.

\section{Ready to submit your research? Choose BMC and benefit from:}

- fast, convenient online submission

- thorough peer review by experienced researchers in your field

- rapid publication on acceptance

- support for research data, including large and complex data types

- gold Open Access which fosters wider collaboration and increased citations

- maximum visibility for your research: over $100 \mathrm{M}$ website views per year

At BMC, research is always in progress.

Learn more biomedcentral.com/submissions 